\title{
First order structure-preserving perturbation theory for eigenvalues of symplectic matrices
}

\author{
Fredy Sosa, Julio Moro* \& Christian Mehl ${ }^{\dagger}$
}

March 20, 2018

\begin{abstract}
A first order perturbation theory for eigenvalues of real or complex $J$-symplectic matrices under structure-preserving perturbations is developed. As main tools structured canonical forms and Lidskii-like formulas for eigenvalues of multiplicative perturbations are used. Explicit formulas, depending only on appropriately normalized left and right eigenvectors, are obtained for the leading terms of asymptotic expansions describing the perturbed eigenvalues. Special attention is given to eigenvalues on the unit circle, especially to the exceptional eigenvalues \pm 1 , whose behavior under structure-preserving perturbations is known to differ significantly from the behavior under general perturbations. Several numerical examples are used to illustrate the asymptotic expansions.
\end{abstract}

\section{Introduction}

The main goal of this paper is to develop a first order perturbation theory for eigenvalues of $J$-symplectic matrices under structure-preserving perturbations.

Definition 1.1 Let $J \in \mathbb{C}^{2 n \times 2 n}$ be an invertible skew-symmetric matrix. A matrix $S \in \mathbb{C}^{2 n \times 2 n}$ is called $J$-symplectic if $S^{T} J S=J$, and $J$-Hamiltonian if $S^{T} J+J S=0$.

The case most relevant for applications is when $S$ is real and $J$ takes the special form

$$
J:=\left[\begin{array}{cc}
0 & I_{n} \\
-I_{n} & 0
\end{array}\right] \in \mathbb{R}^{2 n \times 2 n},
$$

where $I_{n}$ denotes the $n \times n$ identity matrix. Note that this $J$ is not only skew-symmetric, but also orthogonal, i.e., $J^{-1}=J^{T}=-J$. In this special case, $S$ is simply called either symplectic or Hamiltonian rather than $J$-symplectic or J-Hamiltonian.

The situation we analyze is as follows: given a (possibly multiple) eigenvalue $\lambda$ of a $J$-symplectic matrix $S$, we consider another $J$-symplectic matrix $\widehat{S}$ close to $S$, and develop asymptotic expansions of the eigenvalues of $\widehat{S}$ close to $\lambda$ by interpreting $\widehat{S}=\widehat{S}(\varepsilon)$ as a particular value of an analytic $J$-symplectic matrix function $\widehat{S}(\cdot)$ depending on a real parameter $\varepsilon$. The fact that both $S$ and $\widehat{S}$ are $J$-symplectic is crucial: it is well known (see, for instance, $[15,1]$ ) that the eigenvalues of $J$-symplectic matrices behave quite differently under structure-preserving perturbations in comparation to arbitrary perturbations.

The symplectic eigenvalue problem, i.e., the problem of computing eigenvalues, eigenvectors, and invariant subspaces of symplectic matrices is an important one in linear control theory for discrete-time systems. In particular, the symplectic eigenvalue problem plays a major role for the solution of the linear-quadratic optimal control problem or the solution of discrete-time algebraic Riccati equations, see [7] and the references therein. Several algorithms for the solution of the symplectic eigenvalue problem have been proposed, see, e.g. [3, 4,

\footnotetext{
${ }^{*}$ F. Sosa and J. Moro are with the Departamento de Matemáticas, Universidad Carlos III de Madrid, Spain ( $\{$ fsosa, jmoro\}euc $3 \mathrm{~m}$.es). The research of both authors was partially supported by the spanish Ministerio de Economía y Competitividad under grant MTM2014-54692-P

${ }^{\dagger}$ C. Mehl is with the Institut für Mathematik, Sekretariat MA 4-5, Technische Universität Berlin, 10623 Berlin, Germany (mehl@math.tu-berlin.de)
} 
$5,11,16]$. All these approaches have in common that they focus on structure preservation, i.e., in each step of the algorithm the property of the underlying matrix to be symplectic is preserved.

Since many decades, the exploitation of structure has been an important aspect in Numerical Linear Algebra. A particular example are symmetry structures with respect to inner products that typically lead to restrictions on the spectra of the corresponding matrices. In the case of $J$-symplectic matrices (which are orthogonal with respect to the skew-symmetric inner product on $\mathbb{C}^{n}$ induced by $J$ ), it is well known that the eigenvalues of real $J$-symplectic matrices are symmetric with respect to the unit circle, a symmetry that is sometimes referred to as symplectic eigenvalue symmetry. If algorithms for the solution of the corresponding eigenvalue problem are applied that ignore the existing structure, then not only round-off errors may cause the loss of the spectral symmetry, but also other important aspects of the problem may become invisible as additional invariants that only exist under structure-preserving transformations may cause special effects in the perturbation analysis. In the case of $J$-symplectic matrices, one such invariant having a significant impact on the behavior of the spectrum under structure-preserving perturbations is the so-called sign characteristic of unimodular eigenvalues (see (23) below for a formal definition).

To illustrate the important role that the sign characteristic plays in perturbation theory, let us consider the following example. First, recall that stability of discrete systems depends on the location of eigenvalues of the underlying matrix with respect to the unit circle. Thus, a symplectic matrix can be considered to be stable if all eigenvalues are contained in the closed unit disc with those on the unit circle being semisimple. If we consider the two symplectic matrices

$$
S_{1}=\left[\begin{array}{rrrr}
0 & 1 & 0 & 0 \\
-1 & 0 & 0 & 0 \\
0 & 0 & 0 & 1 \\
0 & 0 & -1 & 0
\end{array}\right], \quad S_{2}=\left[\begin{array}{rrrr}
0 & 0 & 1 & 0 \\
0 & 0 & 0 & 1 \\
-1 & 0 & 0 & 0 \\
0 & -1 & 0 & 0
\end{array}\right],
$$

then both have the eigenvalues $\pm i$ with algebraic and geometric multiplicity two, and thus correspond to stable systems. In particular, both have the same Jordan canonical form and hence one may expect both matrices to behave similarly under perturbations. Indeed this is the case when perturbation are applied that ignore the symplectic structure, because arbitrary small perturbations can move eigenvalues to the outside of the unit circle, and will thus make the system unstable. This is illustrated Figure 1, which shows the eigenvalues of 100 random small arbitrary perturbations of $S_{1}$ (left picture) and $S_{2}$ (right picture). These eigenvalues are located in "clouds" around the eigenvalues $\pm i$ of $S_{1}$ and $S_{2}$, respectively, and may be inside or outside the unit disc. The situation is completely different if the system is subject to structure-preserving transformations. Figure 1 depicts the effect of 100 random small perturbations that result again in a symplectic matrix. While again arbitrarily small perturbations may move eigenvalues of $S_{1}$ to the outside of the unit circle (see third picture), the situation is different for the matrix $S_{2}$, because the eigenvalues of all perturbed symplectic matrices remain on the unit circle (see fourth picture). From this point of view, the system given by $S_{2}$ is robustly stable under structure-preserving perturbations while the system given by $S_{1}$ is not. This surprising behavior of unimodular eigenvalues of symplectic matrices has been observed and explained in the literature before, see e.g. [8, 12], and it is caused by the fact that although both matrices have the same Jordan canonical form, they have different sign characteristics (for a more detailed explanation we refer to Section 4 and, especially, to Remark 5.2.) In particular, this example shows that the perturbation theory can be expected to be significantly different if arbitrary versus structure-preserving perturbations are considered.

Driven by the wish for a better understanding of structure-preserving perturbations of symplectic matrices, the effect of generic rank one perturbations on the Jordan structure of $J$-symplectic matrices was analyzed in [15], and it was discussed in [1] how the results can be extended to perturbations of rank $k>1$. It was observed that the preservation of structure sometimes has an important impact on how algebraic and partial multiplicities (i.e., sizes of Jordan blocks) of eigenvalues change under low rank perturbations. In particular, it became apparent that the eigenvalues \pm 1 show an exceptional behavior due to symmetry restrictions in the Jordan canonical forms of symplectic matrices - an effect that cannot be observed if structure is ignored and arbitrary perturbations are applied.

While the change in the Jordan canonical form for a given eigenvalue of a symplectic matrix is now well understood, not much has been said about new eigenvalues, i.e., eigenvalues of the perturbed matrix which were not eigenvalues of the original unperturbed one. Based on the techniques used in [15], it was possible to 

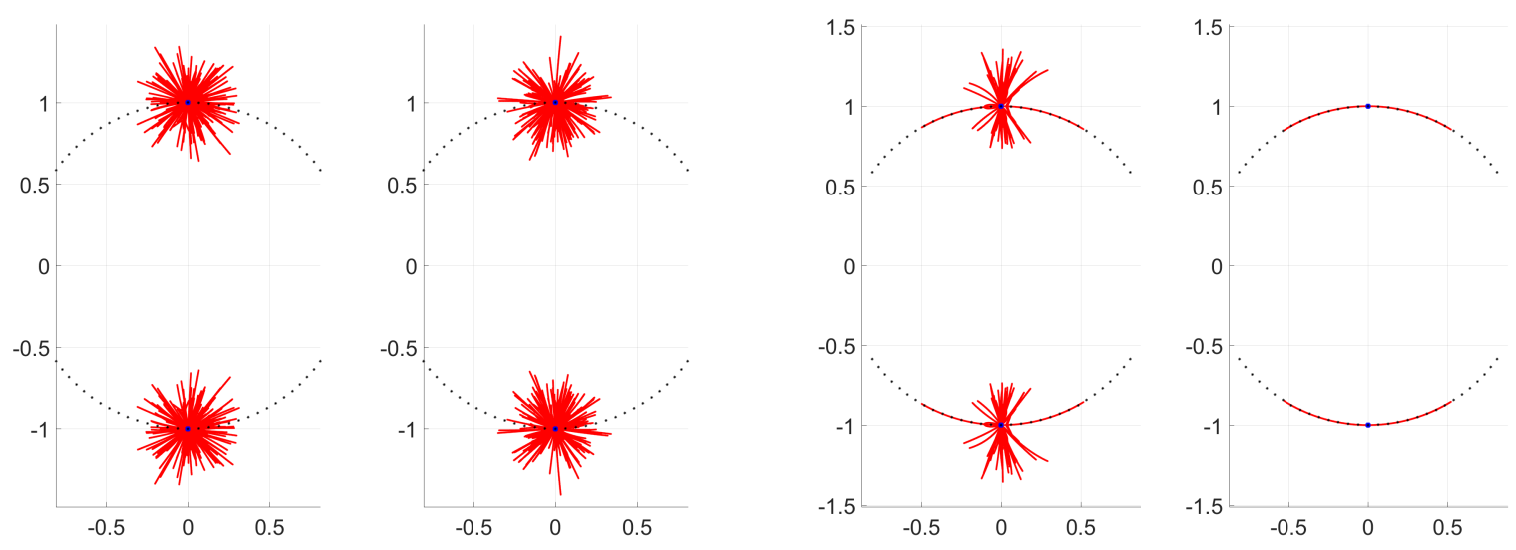

(a) ignoring structure

(b) preserving structure

Figure 1: Structure-preserving vs. structure-ignoring perturbations

show that generically these new eigenvalues will be simple, but only very limited statements on their location in the complex plane could be made. It is the aim of this paper to fill this gap and to investigate the movement of eigenvalues under structure-preserving perturbations depending on a small real parameter $\varepsilon$.

The formulas we obtain rely on two fundamental ingredients, namely (i) the multiplicative spectral perturbation theory described in $\S 2.2$ below and (ii) the detailed analysis performed in $\S 3.3$ of the connection between left and right eigenvectors of $J$-symplectic matrices. It may be worth at this point to highlight the importance of each of these two items:

(i) the choice of a multiplicative approach for the perturbation analysis, as in [20], instead of the usual additive one (see, for instance, [17]), is more natural in this context due to the underlying multiplicativity of symplectic structure ${ }^{1}$ : let $S$ be a $J$-symplectic matrix and let $\widehat{S}(\varepsilon)$ be an analytic $J$-symplectic matrix function in the real parameter $\varepsilon$ with $\widehat{S}(0)=S$. If we write $\widehat{S}(\varepsilon)$ as a multiplicative perturbation

$$
\widehat{S}(\varepsilon)=\left(I+\varepsilon B+O\left(\varepsilon^{2}\right)\right) S,
$$

one can easily check (see $\S 2.1$ below) that the matrix $B$ must be $J$-Hamiltonian, a fact that will be exploited extensively throughout our analysis. This crucial property would be lost in an additive representation

$$
\widehat{S}(\varepsilon)=S+\varepsilon E+O\left(\varepsilon^{2}\right),
$$

where the perturbation matrix $E=B S$ is the product of a $J$-Hamiltonian and a $J$-symplectic matrix, and has therefore no recognizable structure;

(ii) while multiplicative perturbation theory quantitatively accounts for the size of the leading asymptotic terms, it does not provide much geometric information as to the symmetry constraints the perturbed eigenvalues must satisfy (recall that for each value of $\varepsilon$ the perturbed matrix $\widehat{S}(\varepsilon)$ is still $J$-symplectic). Such symmetries are not apparent right away from the expansions, unless one digs deeper into the formulas for the coefficients: the key observation here is that, as shown in Theorem 2.3 below, those formulas involve left and right eigenvectors of the unperturbed matrix, and these vectors are connected in a special way when the matrix is $J$-symplectic. This, together with the special properties of $B$ in (3) as a $J$-Hamiltonian matrix, will allow us to make the symmetries explicit in the formulas.

The main tool to reveal the connections between left and right eigenvectors are structured symplectic canonical forms, described in $\S 3.1$ below: working out how they relate with the Jordan canonical form leads to explicit relationships between appropriately chosen left and right eigenvectors which, in turn, allow further refinement of the formulas obtained via Theorem 2.3, so that spectral symmetries are explicitly shown in the expansions. This is especially important when the unperturbed eigenvalue is

\footnotetext{
${ }^{1}$ If $S_{1}, S_{2}$ are $J$-symplectic, then the sum $S_{1}+S_{2}$ is, in general, not $J$-symplectic, while the matrix product $S_{1} S_{2}$ is.
} 
either of the critical eigenvalues \pm 1 , since we will show that any other eigenvalues behave similarly under structure-preserving and under general perturbations. The exceptional behavior of \pm 1 under structure-preserving perturbations is what will make them the main object of our focus in $\S 4.3$.

The remainder of the paper is organized as follows. Section 2 describes the perturbations we consider and briefly recalls the basic theory for general multiplicative perturbations. Section 3 is devoted to connecting the left and right eigenvectors showing up in the Jordan form (which are crucial for the asymptotic expansions) with vectors appearing in the structured canonical form. This will allow us to reveal the special relationships between left and right eigenvectors induced by symplectic structure. Once these relationships are available, Section 4 presents the asymptotic expansions for eigenvalues of arbitrary structure-preserving $J$-symplectic perturbations obtained by applying the multiplicative perturbation theory in Section 2. Special attention is given to the exceptional eigenvalues \pm 1 whose perturbation behavior is far richer than the one for other eigenvalues, either inside or outside the unit circle. Explicit formulas are obtained for the leading terms in all possible situations, except for a highly nongeneric one, already identified in [15], where the spectral constraints due to symplecticity clash with the typical behavior of Jordan blocks under perturbation. Separate attention is also given to the case of $J$-symplectic perturbations of rank one, which is especially clarifying because of the simplicity of the asymptotic formulas. These formulas highlight the crucial role played by the sign characteristics in the perturbation analysis. In Subsection 4.4 we make use of these formulas pertaining rankone perturbations to make a few observations describing the possible behaviors of eigenvalues on the unit circle which coalesce under the effect of structure-preserving rank-one perturbations. Section 5 illustrates the expansions in Section 4 with several numerical examples. We conclude with the appendix A which describes a kind of auxiliary perturbations used in Section 5 to single out which eigenvalues in the numerical examples are covered by our first-order perturbation theory and which ones are due to higher order terms.

\section{Preliminaries}

\subsection{Small multiplicative $J$-symplectic perturbations}

We start our analysis by showing that, as announced above, any analytic $J$-symplectic matrix function $\widehat{S}(\varepsilon)$ in a real parameter $\varepsilon$ with $\widehat{S}(0)=S$ can be written multiplicatively in the form (3) for some $J$-Hamiltonian matrix $B$ : if $\|\cdot\|$ is any matrix norm then we can write

$$
\frac{\|\widehat{S}(\varepsilon)-S\|}{\|S\|}=O(\varepsilon) .
$$

Since $S$ is $J$-symplectic, and therefore invertible, we have

$$
\frac{\|\widehat{S}(\varepsilon)-S\|}{\|S\|}=\frac{\|\widehat{S}(\varepsilon)-S\|\left\|S^{-1}\right\|}{\kappa(S)} \geq \frac{\left\|\widehat{S}(\varepsilon) S^{-1}-I_{2 n}\right\|}{\kappa(S)},
$$

where $\kappa(S)=\|S\|\left\|S^{-1}\right\|$ is the condition number of $S$ in the norm of choice. Hence,

$$
\left\|\widehat{S}(\varepsilon) S^{-1}-I_{2 n}\right\| \leq \kappa(S) O(\varepsilon)
$$

and consequently the matrix function $\widehat{I}(\varepsilon):=\widehat{S}(\varepsilon) S^{-1}$ satisfies $\widehat{I}(0)=I_{2 n}$ and can be expanded as a power series

$$
\widehat{I}(\varepsilon)=I_{2 n}+\varepsilon B+O\left(\varepsilon^{2}\right)
$$

for some matrix $B \in \mathbb{C}^{2 n \times 2 n}$. Now, recall that the set of $J$-symplectic matrices forms a (Lie) group with respect to matrix multiplication. Hence, $\widehat{I}(\varepsilon)$ is $J$-symplectic which implies

$$
J=\widehat{I}(\varepsilon)^{T} J \widehat{I}(\varepsilon)=J+\varepsilon\left(B^{T} J+J B\right)+O\left(\varepsilon^{2}\right) .
$$

Comparing the coefficients on both sides yields $B^{T} J+J B=0$, i.e., $B$ must be $J$-Hamiltonian. Therefore, any small structure-preserving perturbation $\widehat{S}$ of a $J$-symplectic matrix, can be modelled as

$$
\widehat{S}=\widehat{S}(\varepsilon)=\widehat{I}(\varepsilon) S=\left(I_{2 n}+\varepsilon B+O\left(\varepsilon^{2}\right)\right) S,
$$


where $B$ is $J$-Hamiltonian. Notice that parametrizing the perturbation in terms of $\varepsilon$ as in (3) opens the way for describing the perturbed eigenvalues $\lambda(\varepsilon)$ of $\widehat{S}(\varepsilon)$ through fractional power expansions in $\varepsilon$ (see [10, 2]).

We conclude by observing that any arbitrary $J$-Hamiltonian matrix can take the role of the matrix $B$ in the multiplicative perturbation as in (3). To show this, recall that the matrix $\widehat{I}(\varepsilon)$ in (4) is $J$-symplectic, and its upper left $n \times n$ block is nonsingular for $\varepsilon$ small enough. Thus, we can make use of a characterization due to Dopico \& Johnson of the subset of $2 n \times 2 n$ symplectic matrices with regular upper left $n \times n$ block, namely Theorem 3.1 in [6]. Adapting that result to the particular structure (3) of our perturbations, it is straightforward to prove the following:

Lemma 2.1 Let $S \in \mathbb{C}^{2 n \times 2 n}$ be J-symplectic. Then a complex $2 n \times 2 n$ matrix

$$
B=\left[\begin{array}{ll}
B_{11} & B_{12} \\
B_{21} & B_{22}
\end{array}\right]
$$

partitioned into $n \times n$ blocks, is the first-order coefficient of a J-symplectic analytic matrix function $\widehat{S}(\varepsilon)$ of the form (3) if and only if $B_{22}=-B_{11}^{T}$ and $B_{12}, B_{21}$ are complex symmetric.

This result will also be useful in Section 5 below when generating numerical examples.

\subsection{Lidskii-like formulas for multiplicative perturbations}

Once we have established the multiplicative perturbation framework, our goal in this section is to recall the basic first order perturbation theory for eigenvalues of unstructured multiplicative perturbations, (see [20]). Although for obvious reasons we will set up the notation for a $J$-symplectic unperturbed matrix $S$, Theorem 2.3 below applies to any nonzero eigenvalue of an unperturbed matrix $S, J$-symplectic or not, and to any multiplicative perturbation of $S$ of the form (5), again $J$-symplectic or not.

Let $\lambda$ be an eigenvalue of the $J$-symplectic matrix $S$ and let $S$ have the Jordan canonical form

$$
\left[\begin{array}{c|c}
\mathcal{J} & \\
\hline & \widehat{\mathcal{J}}
\end{array}\right]=\left[\begin{array}{c}
Q \\
\hline \widehat{Q}
\end{array}\right] S[P \mid \widehat{P}]
$$

with

$$
\left[\frac{Q}{\widehat{Q}}\right][P \mid \widehat{P}]=I,
$$

where the Jordan blocks in $\mathcal{J}$ correspond to the (possibly multiple) eigenvalue $\lambda$ of $S$, and $\widehat{\mathcal{J}}$ is the part of the Jordan form containing the eigenvalues of $S$ other than $\lambda$. We take $\mathcal{J}$ to be partitioned as

$$
\mathcal{J}=\operatorname{Diag}\left(\Gamma_{1}^{1}, \ldots, \Gamma_{1}^{r_{1}}, \ldots, \Gamma_{q}^{1}, \ldots, \Gamma_{q}^{r_{q}}\right),
$$

where, for $j=1, \ldots, q$,

$$
\Gamma_{j}^{1}=\ldots=\Gamma_{j}^{r_{j}}=\mathcal{J}_{n_{j}}(\lambda)=\left[\begin{array}{ccccc}
\lambda & 1 & & & \\
& \cdot & \cdot & & \\
& & \cdot & \cdot & \\
& & \cdot & 1 \\
& & & \lambda
\end{array}\right]
$$

is a Jordan block of dimension $n_{j}$, repeated $r_{j}$ times, and ordered so that

$$
n_{1}>n_{2}>\ldots>n_{q} .
$$

Thus, any possible Jordan structure is allowed. Notice that, due to $J$-symplecticity, if $\lambda \notin\{-1,1\}$, then $1 / \lambda$ is also an eigenvalue of $S$ with the same Jordan structure as $\lambda$. Hence, there is the same number of Jordan blocks associated with $\lambda$ as with $1 / \lambda$ for each block size $n_{j}$. 
The matrix $P$ is further partitioned as

$$
P=\left[\begin{array}{l|l|l|l|l|l|l}
P_{1}^{1} & \ldots & P_{1}^{r_{1}} & \ldots & P_{q}^{1} & \ldots & P_{q}^{r_{q}}
\end{array}\right]
$$

conformally with (8). Notice that the columns of each $P_{j}^{k}$ form a right Jordan chain for $S$ of length $n_{j}$ corresponding to $\lambda$. If we denote by $x_{j}^{k}$ the first column of $P_{j}^{k}$, each $x_{j}^{k}$ is a right eigenvector of $S$ associated with $\lambda$. Analogously, if we split

$$
Q=\left[\left(Q_{1}^{1}\right)^{T}|\ldots|\left(Q_{1}^{r_{1}}\right)^{T}|\ldots|\left(Q_{q}^{1}\right)^{T}|\ldots|\left(Q_{q}^{r_{q}}\right)^{T}\right]^{T},
$$

also conformally with (8), the rows of each $Q_{j}^{k}$ form a left Jordan chain of $S$ of length $n_{j}$ corresponding to $\lambda$ and, if we denote by $y_{j}^{k}$ the last (i.e. $n_{j}-$ th) row of $Q_{j}^{k}$, then each $y_{j}^{k}$ is a left eigenvector corresponding to $\lambda$. With these eigenvectors we build up matrices

$$
Y_{j}=\left[\begin{array}{c}
y_{j}^{1} \\
\vdots \\
y_{j}^{r_{j}}
\end{array}\right], \quad X_{j}=\left[x_{j}^{1}, \ldots, x_{j}^{r_{j}}\right]
$$

for $j=1, \ldots, q$, and

$$
W_{s}=\left[\begin{array}{c}
Y_{1} \\
\vdots \\
Y_{s}
\end{array}\right], \quad Z_{s}=\left[X_{1}, \ldots, X_{s}\right]
$$

for $s=1, \ldots, q$.

Finally, given any arbitrary matrix $K \in \mathbb{C}^{2 n \times 2 n}$, we define associated square matrices $\Phi_{s}(K)$ and $E_{s}$ of dimension

$$
f_{s}=\sum_{j=1}^{s} r_{j}
$$

by

$$
\begin{aligned}
& \Phi_{s}(K)=W_{s} K Z_{s}, \quad s=1, \ldots, q, \\
& E_{1}=I, \quad E_{s}=\left[\begin{array}{ll}
0 & 0 \\
0 & I
\end{array}\right] \quad \text { for } s=2, \ldots, q,
\end{aligned}
$$

where the identity block in $E_{s}$ has dimension $r_{s}$. Note that, due to the cumulative definitions of $W_{s}$ and $Z_{s}$, every $\Phi_{s-1}(K)$ is the upper left block of $\Phi_{s}(K)$ for $s=2, \ldots, q$. This nested structure allows us to define the Schur complement $\left(\Phi_{s} / \Phi_{s-1}\right)(K)$ of $\Phi_{s-1}(K)$ in $\Phi_{s}(K)$ whenever $\Phi_{s-1}(K)$ is nonsingular. For each $s>1$, we have

$$
\Phi_{s}(K)=\left[\begin{array}{c|c}
\Phi_{s-1}(K) & W_{s-1} K X_{s} \\
\hline Y_{s} K Z_{s-1} & Y_{s} K X_{s}
\end{array}\right]
$$

and, consequently, the Schur complement is defined as

$$
\left(\Phi_{s} / \Phi_{s-1}\right)(K)=Y_{s}\left(K-K Z_{s-1}\left(\Phi_{s-1}(K)\right)^{-1} W_{s-1} K\right) X_{s} .
$$

Remark 2.2 As a consequence of the partitions above, we have

$$
Q_{j}^{k} S P_{j}^{k}=\Gamma_{j}^{k}=\mathcal{J}_{n_{j}}(\lambda)
$$

for every $j=1, \ldots, q ; k=1, \ldots, r_{j}$. 
We are now in the position to state the following multiplicative perturbation result for nonzero ${ }^{2}$ unperturbed eigenvalues:

Theorem 2.3 ([20]) Let $\lambda$ be an eigenvalue of a complex $n \times n$ matrix $S$ with Jordan structure (6), and let $B$ be an arbitrary $n \times n$ complex matrix. Let $j \in\{1, \ldots, q\}$ be given and assume that $\Phi_{j-1}(B)$ is nonsingular if $j>1$, where $\Phi_{j-1}(\cdot)$ is defined as in (11). Then there are $r_{j} n_{j}$ eigenvalues of the perturbed matrix $\widehat{S}(\varepsilon)=\left(I+\varepsilon B+O\left(\varepsilon^{2}\right)\right) S$ admitting first order expansions

$$
\widehat{\lambda}_{j, k, l}(\varepsilon)=\lambda+\left(\lambda \xi_{j, k}\right)^{1 / n_{j}} \varepsilon^{1 / n_{j}}+o\left(\varepsilon^{1 / n_{j}}\right),
$$

where

(i) the $\xi_{j, k}, k=1, \ldots, r_{j}$, are the roots of the equation

$$
\operatorname{det}\left(\Phi_{j}(B)-\xi E_{j}\right)=0
$$

with $\Phi_{j}$ and $E_{j}$ as in (11). Equivalently, the $\xi_{j, k}, k=1, \ldots, r_{j}$, are the eigenvalues of the Schur complement $\left(\Phi_{j} / \Phi_{j-1}\right)(B)$ of $\Phi_{j-1}(B)$ in $\Phi_{j}(B)$ (if $j=1$, the $\xi_{1, k}$ are just the $r_{1}$ eigenvalues of $\Phi_{1}(B)$ );

(ii) the values $\hat{\lambda}_{j, k, l}(\varepsilon)$ for $l=1, \ldots, n_{j}$ are defined by taking the $n_{j}$-th roots of $\xi_{j, k}$ (those are pairwise distinct unless $\left.\xi_{j, k}=0\right)$.

Several observations are in order about Theorem 2.3 at this point:

Remark 2.4 Theorem 2.3 only speaks of the perturbed eigenvalues with lowest possible leading exponent, i.e., the ones moving fastest away from $\lambda$, which are the ones depending only on the first-order coefficient matrix $B$. Of course the $O\left(\varepsilon^{2}\right)$ terms in (3) may increase the rank of the perturbation $\widehat{S}(\varepsilon)-S$, and consequently destroy further Jordan blocks of $S$, which gives rise to other perturbed eigenvalues, not covered by Theorem 2.3. But these eigenvalues will have leading exponents larger than $1 / n_{j}$, and will be left out of our analysis. Section 5 below provides many instances when this happens.

Remark 2.5 Theorem 2.3 does not say that $\widehat{S}$ has exactly $r_{j} n_{j}$ eigenvalues of order $\varepsilon^{1 / n_{j}}$. This only happens if all $r_{j}$ eigenvalues of the Schur complement $\left(\Phi_{j} / \Phi_{j-1}\right)(B)$ are nonzero. To be more precise, let $r=\operatorname{rank}(B)$. Then there exist two positive integers $j \in\{1, \ldots, q\}$ and $\rho \in\left\{1, \ldots, r_{j}\right\}$ such that

$$
r=\sum_{k=1}^{j-1} r_{k}+\rho=f_{j-1}+\rho
$$

where $f_{j-1}$ is as defined in (10). Then, for every index $i \in\{1, \ldots, q\}, i<j$, Theorem 2.3 implies that there will be generically $r_{i} n_{i}$ eigenvalues of order $\varepsilon^{1 / n_{i}}$ generated by the destruction of all $r_{i}$ Jordan blocks of size $n_{i}$. For the index $j$, however, at most $\rho n_{j}$ eigenvalues of order $\varepsilon^{1 / n_{j}}$ may exist in $\widehat{S}$ if $\Phi_{j-1}(B)$ is nonsingular, since $\operatorname{rank} \Phi_{j-1}(B)=f_{j-1}=r-\rho$ and, therefore,

$$
\operatorname{rank}\left(\Phi_{j} / \Phi_{j-1}\right)(B)=\operatorname{rank} \Phi_{j}(B)-\operatorname{rank} \Phi_{j-1}(B) \leq \rho,
$$

i.e., the Schur complement $\left(\Phi_{j} / \Phi_{j-1}\right)(B)$ may have at most $\rho$ nonzero eigenvalues. For each of these nonzero eigenvalues, formula (13) provides $n_{j}$ different expansions, one for each $n_{j}$-th root. Notice that the expansions (13) still formally hold for all perturbed eigenvalues $\widehat{\lambda}_{j, k, l}(\varepsilon)$, but they provide no information whatsoever for the $\left(r_{j}-\rho\right) n_{j}$ perturbed eigenvalues corresponding to the values $\xi_{j, k}$ that are zero (other than the fact that the corresponding perturbed eigenvalues are of order $\varepsilon^{q}$ for some $q$ larger than $1 / n_{j}$ ). As to the indices $i>j$, Theorem 2.3 does not apply, since every $\Phi_{i-1}$ is singular.

\footnotetext{
${ }^{2}$ If $\lambda=0$ in the statement of Theorem 2.3, the result is trivially true, but gives no information whatsoever on the leading terms. Recall that $J$-symplectic matrices have only nonzero eigenvalues.
} 
Remark 2.6 As long as it corresponds to a nonzero eigenvalue $\xi_{j, k}$ of the Schur complement, the leading term in the expansion (13) is the same for all perturbations (3) having the same first order matrix coefficient $B$. More specifically, let $\widehat{S}(\varepsilon)$ and $\widetilde{S}(\varepsilon)$ be two different $J$-symplectic perturbations of the form (3) with the same first order $J$-Hamiltonian coefficient $B$, and let the common Schur complement $\left(\Phi_{j} / \Phi_{j-1}\right)(B)$ have $\rho$ nonzero eigenvalues. Then, Theorem 2.3 ensures that both $\widehat{S}(\varepsilon)$ and $\widetilde{S}(\varepsilon)$ have $\rho n_{j}$ eigenvalues of order $\varepsilon^{1 / n_{j}}$ with identical leading coefficients. The remaining $\left(r_{j}-\rho\right) n_{j}$ eigenvalues of $\widehat{S}(\varepsilon)$ and $\widetilde{S}(\varepsilon)$ may differ in their leading coefficients, or even in their leading exponents. It may be even that the number of Jordan blocks destroyed by $\widehat{S}(\varepsilon)$ and by $\widetilde{S}(\varepsilon)$ is different, since the ranks of $\widehat{S}-S$ and of $\widetilde{S}-S$ may be different. For both perturbations, however, at least $\rho$ Jordan blocks of size $n_{j}$ will be destroyed, and the $\rho n_{j}$ corresponding eigenvalues will display the very same first order behavior, described by Theorem 2.3, for both perturbations. This observation will be relevant in Section 5 below, where we will introduce such an auxiliary perturbation $\widetilde{S}$ matrix in order to single out from the numerical examples those eigenvalues which conform to the expansions obtained in Section 4.

\section{Jordan chains for symplectic matrices}

Theorem 2.3 shows that the leading coefficients we are looking for are determined by the matrices $\Phi_{j}(B)$ defined in (11), and these largely depend on left and right eigenvectors of $S$ associated with $\lambda$. Notice that the choice of these left and all right eigenvectors is not arbitrary: first, they are implicitly normalized by the fact that they show up in the same Jordan canonical form. Our goal in this section is to show that we may further narrow the choice of the Jordan vectors in such a way that a special connection, induced by symplecticity, is revealed between left and right eigenvectors. Our main auxiliary tools to do so will be structured canonical forms for $J$-symplectic matrices (see [13] and [14] for more details).

\subsection{Real and complex symplectic canonical forms}

Here we briefly review the three different symplectic structured canonical forms we shall be using to extract Jordan vectors. In order to describe them, we need to introduce some auxiliary matrices: for each positive integer $n \in \mathbb{N}$, we define matrices $\Sigma_{n}$ and $R_{n}$ as

$$
\Sigma_{n}=\left[\begin{array}{ccc}
0 & & (-1)^{0} \\
& . & \\
(-1)^{n-1} & & 0
\end{array}\right], \quad R_{n}=\left[\begin{array}{ccc}
0 & 1 \\
& . \cdot & \\
1 & & 0
\end{array}\right]
$$

Furthermore, if $a=\left[\begin{array}{lll}a_{1} & \ldots & a_{n}\end{array}\right]^{T} \in \mathbb{C}^{n}$, then we denote by $\operatorname{Toep}\left(a_{1}, \ldots, a_{n}\right)$ the upper triangular Toeplitz matrix that has $a^{T}$ as its first row.

In the most general case, when all we know is that the unperturbed $J$-symplectic matrix $S$ is complex, we shall use the complex canonical form in Theorem 8.5 of [13], which can be written as follows:

Theorem 3.1 (Theorem 8.5 in [13]) Let $S \in \mathbb{C}^{2 n \times 2 n}$ be a J-symplectic matrix. Then there exists a nonsingular matrix $\mathcal{T}$ such that

$$
\mathcal{T}^{-1} S \mathcal{T}=S_{1} \oplus \ldots \oplus S_{p}, \quad \mathcal{T}^{T} J \mathcal{T}=H_{1} \oplus \ldots \oplus H_{p}
$$

where $S_{j}$ and $H_{j}$ have one of the following forms:

i) even sized blocks associated with $\lambda_{j}= \pm 1$, where $n_{j} \in \mathbb{N}$ is even:

$$
S_{j}=\operatorname{Toep}\left(\lambda_{j}, 1, t_{2}, \ldots, t_{n_{j}-1}\right), \quad H_{j}=\Sigma_{n_{j}},
$$

where the parameters $t_{k}$ are real and uniquely determined by the recursive formula

$$
t_{2}=\frac{1}{2}, \quad t_{2 \ell+1}=0, \quad t_{2 \ell+2}=-\frac{1}{2} \sum_{\nu=1}^{\ell} t_{2 \nu} t_{2(\ell+1-\nu)}, \quad \ell \geq 1
$$


ii) paired blocks associated with $\lambda_{j}= \pm 1$, where $n_{j} \in \mathbb{N}$ is odd:

$$
S_{j}=\left[\begin{array}{c|c}
\mathcal{J}_{n_{j}}\left(\lambda_{j}\right) & \\
\hline & \mathcal{J}_{n_{j}}\left(\lambda_{j}\right)^{-T}
\end{array}\right], \quad H_{j}=\left[\begin{array}{c|c}
0 & I_{n_{j}} \\
\hline-I_{n_{j}} & 0
\end{array}\right] ;
$$

iii) blocks associated with the pair $\left(\lambda_{j}, \lambda_{j}^{-1}\right) \in \mathbb{C} \times \mathbb{C}$, satisfying $\operatorname{Re}\left(\lambda_{j}\right)>\operatorname{Re}\left(\lambda_{j}^{-1}\right)$, or $\operatorname{Im}\left(\lambda_{j}\right)>$ $\operatorname{Im}\left(\lambda_{j}^{-1}\right)$ if $\boldsymbol{\operatorname { R e }}\left(\lambda_{j}\right)=\boldsymbol{\operatorname { R e }}\left(\lambda_{j}^{-1}\right)$, where $n_{j} \in \mathbb{N}$ :

$$
S_{j}=\left[\begin{array}{l|l}
\mathcal{J}_{n_{j}}\left(\lambda_{j}\right) & \\
\hline & \mathcal{J}_{n_{j}}\left(\lambda_{j}\right)^{-T}
\end{array}\right], \quad H_{j}=\left[\begin{array}{c|c}
0 & I_{n_{j}} \\
\hline-I_{n_{j}} & 0
\end{array}\right] .
$$

Remark 3.2 The pairing in part (ii) shows that for either of the critical eigenvalues \pm 1 the number of oddsized Jordan blocks is always even.

It is important to remark here that the second equation in (17) can also be written as

$$
\mathcal{T}^{T} J=\left(H_{1} \oplus \ldots \oplus H_{p}\right) \mathcal{T}^{-1},
$$

which is sometimes more convenient, as we will see later.

If $S$ is a real symplectic matrix, then we may also consider the canonical form in Theorem 5.5 of [14] to get the Jordan chains associated with real eigenvalues. The only difference with the canonical form in Theorem 3.1 is that now $\mathcal{T} \in \mathbb{R}^{2 n \times 2 n}$, and thus Jordan chains associated with real eigenvalues obtained from the symplectic canonical form have real entries. For each even-sized block corresponding to $\lambda_{j}= \pm 1$, a sign $\varsigma_{j}$ appears in equation (18) for $H_{j}$,

$$
H_{j}=\varsigma_{j} \Sigma_{n_{j}}, \quad \varsigma_{j}= \pm 1
$$

which is called the sign associated with that Jordan block. The list of all signs corresponding to Jordan blocks associated with $\lambda_{j}$ is called the sign characteristic of $\lambda_{j}$.

The case when $S \in \mathbb{R}^{2 n \times 2 n}$ and the eigenvalue $\lambda$ lies on the unit circle is special: in this case it will be advantageous to consider the real matrix $S$ as a complex matrix which turns out to be $(i J)$-unitary, i.e., which satisfies $S^{*}(i J) S=i J$. This allows us to make use of the structured canonical form for $H$-unitary matrices for Hermitian $H$ (see Theorem 6.6 of [13]). This canonical form distinguishes two cases, just like Theorem 3.1, depending on whether the eigenvalue lies on the unit circle or not. Since we will be only interested in unimodular eigenvalues (for the others, the two previous canonical forms already give better results), we need only describe the blocks $S_{j}$ and $H_{j}$ associated with $\lambda_{j}$ such that $\left|\lambda_{j}\right|=1$, namely,

$$
S_{j}=\operatorname{Toep}\left(\lambda_{j}, i \lambda_{j},-i \lambda_{j} t_{2}, \ldots,-i \lambda_{j} t_{n_{j}-1}\right), \quad H_{j}=\varsigma_{j} R_{n_{j}}, \quad \varsigma_{j}= \pm 1,
$$

where the parameters $t_{k}$ are as in (19). It should be noted that even though this is also true for $\lambda= \pm 1$, for these two particular eigenvalues it is more advantageous to use the real canonical form, since it provides more detailed information for odd-sized blocks, and it also takes into account the fact that they appear in pairs in the canonical form.

\subsection{Extracting Jordan bases from bases associated with symplectic canonical forms}

Although the structured canonical forms from $\S 3.1$ are closely related to the Jordan form, they do not directly display the left and right eigenvectors needed to apply Theorem 2.3. This is why we need to find appropriate changes of basis which similarity-transform the structured symplectic form into the Jordan form. These transformations will reveal the connections induced by the symplectic structure between certain left and right eigenvectors, which will in turn allow us to write more detailed formulas for the leading terms in the asymptotic expansions. 
- Let $\lambda \in \mathbb{C} \backslash\{0\}$, let $p$ be a positive integer and let $\mathcal{J}_{p}(\lambda)$ be an upper triangular Jordan block of size $p$ with eigenvalue $\lambda$. Then there exists a matrix $D_{p}$ such that

$$
D_{p}^{-1} \mathcal{J}_{p}(\lambda)^{-T} D_{p}=\mathcal{J}_{p}\left(\lambda^{-1}\right)
$$

For $p=2,3,4$ the matrix $D_{p}$ can be chosen as

$$
D_{2}=\left[\begin{array}{cc}
0 & -\lambda^{2} \\
1 & 0
\end{array}\right], \quad D_{3}=\left[\begin{array}{ccc}
0 & 0 & \lambda^{4} \\
\mathbf{0} & -\lambda^{2} & \lambda^{3} \\
\mathbf{1} & \mathbf{0} & 0
\end{array}\right], \quad D_{4}=\left[\begin{array}{cccc}
0 & 0 & 0 & -\lambda^{6} \\
\mathbf{0} & \mathbf{0} & \lambda^{4} & -2 \lambda^{5} \\
\mathbf{0} & -\lambda^{\mathbf{2}} & \lambda^{\mathbf{3}} & \lambda^{4} \\
\mathbf{1} & \mathbf{0} & \mathbf{0} & 0
\end{array}\right]
$$

In general, one can prove that the following recursive sequence provides us with the required change of basis:

$$
D_{1}=[1], \quad D_{p+1}=\left[\begin{array}{c|c}
0_{1 \times p} & \left(-\lambda^{2}\right)^{p} \\
\hline D_{p} & d_{p}
\end{array}\right], \quad p=1,2,3, \ldots,
$$

with $d_{p} \in \mathbb{C}^{p}$ being an appropriate vector of the form $d_{p}=\left[\begin{array}{llll}* & \ldots & * & 0\end{array}\right]^{T}$. In order to prove this by induction, note first that $(25)$ is equivalent to

$$
\mathcal{J}_{p}(\lambda)^{T} D_{p} \mathcal{J}_{p}\left(\lambda^{-1}\right)=D_{p}
$$

which clearly holds for $p=1$. We now state the induction hypothesis: assume that (27) holds for some $p \in \mathbb{N}$ and $D_{p}$ as in (26). We will show that this implies

$$
\mathcal{J}_{p+1}(\lambda)^{T} D_{p+1} \mathcal{J}_{p+1}\left(\lambda^{-1}\right)=D_{p+1} .
$$

with $D_{p+1}$ as in (26). First, partition the matrices above as

$$
\mathcal{J}_{p+1}(\lambda)^{T}=\left[\begin{array}{c|c}
\lambda & 0_{1 \times p} \\
\hline e_{1} & \mathcal{J}_{p}(\lambda)^{T}
\end{array}\right] \quad \mathcal{J}_{p+1}\left(\lambda^{-1}\right)=\left[\begin{array}{c|c}
\mathcal{J}_{p}\left(\lambda^{-1}\right) & e_{p} \\
\hline 0_{1 \times p} & \lambda^{-1}
\end{array}\right]
$$

where $e_{1}$ and $e_{p}$ are the first and last column, respectively, of the $p \times p$ identity matrix. If we plug these block matrices into (28), we get

$\mathcal{J}_{p+1}(\lambda)^{T} D_{p+1} \mathcal{J}_{p+1}\left(\lambda^{-1}\right)=\left[\begin{array}{c|c}0_{1 \times p} & (-1)^{p} \lambda^{2 p} \\ \hline \mathcal{J}_{p}(\lambda)^{T} D_{p} \mathcal{J}_{p}\left(\lambda^{-1}\right) & \mathcal{J}_{p}(\lambda)^{T}\left(D_{p} e_{p}+\lambda^{-1} d_{p}\right)+(-1)^{p} \lambda^{2 p-1} e_{1}\end{array}\right]$.

Now, since $\mathcal{J}_{p}(\lambda)^{T} D_{p} \mathcal{J}_{p}\left(\lambda^{-1}\right)=D_{p}$ by the induction hypothesis, we only need to prove the existence of a vector $d_{p}$ satisfying

$$
\mathcal{J}_{p}(\lambda)^{T}\left(D_{p} e_{p}+\lambda^{-1} d_{p}\right)+(-1)^{p} \lambda^{2 p-1} e_{1}=d_{p}
$$

or, equivalently,

$$
\left(\lambda^{-1} \mathcal{J}_{p}(\lambda)^{T}-I_{p}\right) d_{p}=(-1)^{p-1} \lambda^{2 p-1} e_{1}-\mathcal{J}_{p}(\lambda)^{T} D_{p} e_{p} .
$$

However, since $D_{p} e_{p}=\left[\left(-\lambda^{2}\right)^{p}, *, \cdots, *\right]^{T}$, we have

$$
\mathcal{J}_{p}(\lambda)^{T} D_{p} e_{p}=\left[\begin{array}{c}
\lambda\left(-\lambda^{2}\right)^{p} \\
* \\
\vdots \\
*
\end{array}\right] \Rightarrow(-1)^{p-1} \lambda^{p-1} e_{1}-\mathcal{J}_{p}(\lambda)^{T} D_{p} e_{p}=\left[\begin{array}{c}
0 \\
* \\
\vdots \\
*
\end{array}\right] .
$$


On the other hand, the first row of $\lambda^{-1} \mathcal{J}_{p}(\lambda)^{T}-I_{p}$ is identically zero, so the subspace generated by its columns is $(p-1)$-dimensional. Therefore, the vector $(-1)^{p-1} \lambda^{p-1} e_{1}-\mathcal{J}_{p}(\lambda)^{T} D_{p} e_{p}$ is in the column space of $\lambda^{-1} \mathcal{J}_{p}(\lambda)^{T}-I_{p}$, which proves the existence of the vector solution $d_{p}$ of (29). It only remains to prove that the last entry of $d_{p}$ can be chosen to be zero, but since the last column of $\lambda^{-1} \mathcal{J}_{p}(\lambda)^{T}-I_{p}$ is identically zero, the last entry of $d_{p}$ is a free variable, and can thus be chosen to be zero.

- Another type of blocks showing up in the symplectic canonical forms are upper triangular Toeplitz matrices of the form

$$
T_{p}=\operatorname{Toep}\left(\lambda, t_{1}, t_{2}, \ldots, t_{p-1}\right), \quad t_{1} \neq 0 .
$$

As before, we need a matrix $A_{p}$ such that

$$
A_{p}^{-1} T_{p} A_{p}=\mathcal{J}_{p}(\lambda)
$$

One can check that, for $p=2,3,4$ the matrices $A_{p}$

$$
A_{2}=\left[\begin{array}{cc}
1 & 0 \\
0 & \frac{1}{t_{1}}
\end{array}\right], \quad A_{3}=\left[\begin{array}{ccc}
\mathbf{1} & \mathbf{0} & 0 \\
\mathbf{0} & \frac{\mathbf{1}}{\mathbf{t}_{1}} & -\frac{t_{2}}{\left(t_{1}\right)^{3}} \\
0 & 0 & \frac{1}{\left(t_{1}\right)^{2}}
\end{array}\right], \quad A_{4}=\left[\begin{array}{cccc}
\mathbf{1} & \mathbf{0} & \mathbf{0} & 0 \\
\mathbf{0} & \frac{\mathbf{1}}{\mathbf{t}_{\mathbf{1}}} & -\frac{\mathbf{t}_{2}}{\left(\mathbf{t}_{1}\right)^{3}} & \frac{2\left(t_{2}\right)^{2}-t_{1} t_{3}}{\left(t_{1}\right)^{5}} \\
\mathbf{0} & \mathbf{0} & \frac{\mathbf{1}}{\left(\mathbf{t}_{1}\right)^{2}} & -\frac{2 t_{2}}{\left(t_{1}\right)^{4}} \\
0 & 0 & 0 & \frac{1}{\left(t_{1}\right)^{3}}
\end{array}\right]
$$

satisfy the equation above. Again, the nested structure in these matrices suggests the recursive formula

$$
\begin{aligned}
A_{1} & =[1] \\
A_{p+1} & =\left[\begin{array}{c|c}
A_{p} & a_{p} \\
\hline 0_{1 \times p} & \left(t_{1}\right)^{-p}
\end{array}\right], \quad p=1,2,3, \ldots
\end{aligned}
$$

for an appropriate vector $a_{p} \in \mathbb{C}^{p}$. Let us prove, again by induction, that these matrices do the job: obviously, $A_{1}$ satisfies $A_{1}^{-1} T_{1} A_{1}=\mathcal{J}_{1}(\lambda)$. Next, assume that

$$
A_{p}^{-1} T_{p} A_{p}=\mathcal{J}_{p}(\lambda)
$$

for some $p \in \mathbb{N}$ and $A_{p}$ as defined in (30). We will prove that for some appropriate $a_{p}$ the matrix $A_{p+1}$ satisfies the corresponding equation with $p$ replaced by $p+1$. If we partition conformally all matrices involved, we obtain using the abbreviation $t=\left[\begin{array}{lll}t_{p-1} & \ldots & t_{1}\end{array}\right]^{T}$ that

$$
\begin{aligned}
T_{p+1} A_{p+1} & =\left[\begin{array}{c|c}
T_{p} & t \\
\hline 0_{1 \times p} & \lambda
\end{array}\right]\left[\begin{array}{c|c}
A_{p} & a_{p} \\
\hline 0_{1 \times p} & \left(t_{1}\right)^{-p}
\end{array}\right]=\left[\begin{array}{c|c}
T_{p} A_{p} & T_{p} a_{p}+\left(t_{1}\right)^{-p} t \\
\hline 0_{1 \times p} & \lambda\left(t_{1}\right)^{-p}
\end{array}\right] \\
A_{p+1} \mathcal{J}_{p+1}(\lambda) & =\left[\begin{array}{c|c}
A_{p} & a_{p} \\
\hline 0_{1 \times p} & \left(t_{1}\right)^{-p}
\end{array}\right]\left[\begin{array}{c|c}
\mathcal{J}_{p}(\lambda) & e_{p} \\
\hline 0_{1 \times p} & \lambda
\end{array}\right]=\left[\begin{array}{cc|c}
A_{p} \mathcal{J}_{p}(\lambda) & A_{p} e_{p}+\lambda a_{p} \\
\hline 0_{1 \times p} & \lambda\left(t_{1}\right)^{-p}
\end{array}\right]
\end{aligned}
$$

The induction hypothesis leads to $T_{p} A_{p}=A_{p} \mathcal{J}_{p}(\lambda)$, so we only need to prove that there exists a vector $a_{p} \in \mathbb{C}^{p}$ such that

$$
T_{p} a_{p}+\left(t_{1}\right)^{-p} t=A_{p} e_{p}+\lambda a_{p}, \quad t=\left[t_{p} t_{p-1} \ldots t_{1}\right]^{T}
$$

or, equivalently,

$$
\left(T_{p}-\lambda I_{p}\right) a_{p}=A_{p} e_{p}-\left(t_{1}\right)^{-p} t
$$


Now, since $t_{1} \neq 0$ and the last row of $T_{p}-\lambda I_{p}$ is identically zero, the vector $A_{p} e_{p}-\left(t_{1}\right)^{-p} t$ is in the column space of $T_{p}-\lambda I_{p}$ if and only if its last entry is zero. But

$$
A_{p} e_{p}-\left(t_{1}\right)^{-p} t=\left[\begin{array}{c}
* \\
\vdots \\
* \\
\left(t_{1}\right)^{-p+1}
\end{array}\right]-\left[\begin{array}{c}
t_{p}\left(t_{1}\right)^{-p} \\
\vdots \\
t_{2}\left(t_{1}\right)^{-p} \\
t_{1}\left(t_{1}\right)^{-p}
\end{array}\right]=\left[\begin{array}{c}
* \\
\vdots \\
* \\
0
\end{array}\right],
$$

which completes the proof.

\subsection{Relationships between left and right Jordan chains}

It is well known that if $x \in \mathbb{C}^{2 n}$ is a right eigenvector associated with the eigenvalue $\lambda \in \mathbb{C} \backslash\{0\}$ of the $J$-symplectic matrix $S$, then $y=x^{T} J$ is a left eigenvector of $S$ associated with the eigenvalue $\lambda^{-1}$. Our aim in this subsection is to extend this result to more general contexts: in $\S 3.2$ we have identified changes of basis allowing us to extract Jordan vectors from vectors in the structured symplectic canonical form. We will now exploit such transformations in order to establish the connections induced by symplectic structure between those left and right eigenvectors constructed starting from the structured canonical form.

To begin with, we partition the structured symplectic canonical form

$$
\left[\begin{array}{c|c}
\mathcal{C} & \\
\hline & \widehat{\mathcal{C}}
\end{array}\right]=\left[\begin{array}{c}
U \\
\hline \widehat{U}
\end{array}\right] S[T \mid \widehat{T}]
$$

in a way similar to (6)-(8), i.e., $\mathcal{C}$ contains all blocks in the canonical form associated with the eigenvalue $\lambda$, while $\widehat{\mathcal{C}}$ contains those corresponding to the remaining eigenvalues. As above, $T$ and $U$ are partitioned into column blocks and row blocks, respectively, conformally with the block structure of $\mathcal{C}$. We denote by $T_{j}^{k}$ the $k$-th block of columns taken from $T$, associated with the $k$-th block of size $n_{j}$ in $\mathcal{C}$ (resp., by $U_{j}^{k}$ the $k$-th block of rows taken from $U$ associated with the $k$-th block of size $n_{j}$ in $\mathcal{C}$ ). Then we have for each $j=1, \ldots, q$ and $k=1, \ldots, r_{j}$ that

$$
U_{j}^{k} S T_{j}^{k}=C_{j}^{k}
$$

where $C_{j}^{k}$ is a block of size $n_{j}$ associated with $\lambda$ in the canonical form $\mathcal{C}$. We distinguish two cases in the analysis, depending on whether $\lambda= \pm 1$ or not. Since the former case is by far the most interesting one, we shall mostly focus on it, although for the sake of completeness we will also report the relationships for eigenvalues other than \pm 1 .

\subsubsection{Case 1: $\lambda \in\{-1,1\}$}

Again, we split the analysis into two different cases, depending on the parity of the block size $n_{j}$ of the blocks $C_{j}^{k}$ in the canonical form:

- Case 1.a: $\lambda \in\{-1,1\}, n_{j}$ even.

If the size $n_{j}$ of $C_{j}^{k}$ is even, then from the first relation in (18) we have that $C_{j}^{k}$ is an upper triangular Toeplitz matrix of the form

$$
\operatorname{Toep}\left(\lambda, 1, t_{2}, \cdots, t_{n_{j}-1}\right) .
$$

Although this matrix is not a Jordan block, it is similar to $\mathcal{J}_{n_{j}}(\lambda)$. Moreover, its structure allows us to conclude that the first column of $T_{j}^{k}$ is a right eigenvector associated with $\lambda$, and the last row of $U_{j}^{k}$ is a corresponding left eigenvector. Furthermore, the nonsingular matrix $A_{n_{j}}$, as defined in (30), is such that

$$
A_{n_{j}}^{-1} \operatorname{Toep}\left(\lambda, 1, t_{2}, \cdots, t_{n_{j}-1}\right) A_{n_{j}}=\mathcal{J}_{n_{j}}(\lambda)
$$


so the left and right Jordan chains associated with this block are, respectively, the columns of

$$
\begin{aligned}
P_{j}^{k} & =U_{j}^{k} A_{n_{j}}, \\
Q_{j}^{k} & =\left(A_{n_{j}}\right)^{-1} T_{j}^{k} .
\end{aligned}
$$

Now, recall that the first column of $A_{n_{j}}$ (resp., the last row of $A_{n_{j}}^{-1}$ ) is the first column $e_{1} \in \mathbb{R}^{n_{j}}$ (resp., the last row $e_{n_{j}}^{T}$ ) of the identity matrix. Hence, the left and right eigenvectors associated with $\lambda$ for this Jordan block are the first column of $U_{j}^{k}$ and the last row of $T_{j}^{k}$, respectively.

Next, recall that we have denoted $\mathcal{T}=[T \mid \widehat{T}]$, and its inverse by

$$
\mathcal{T}^{-1}=\left[\frac{U}{\widehat{U}}\right]
$$

Making use of the congruence relationship (22) and the second equation in (18), we get

$$
\left(T_{j}^{k}\right)^{T} J=\Sigma_{n_{j}} U_{j}^{k}, \quad j=1, \ldots, q, \quad k=1, \ldots, r_{j},
$$

with $\Sigma_{n_{j}}$ given by (16). If, as before, we denote by $y_{j}^{k}$ the last row of $U_{j}^{k}$, and by $x_{j}^{k}$ the first column of $T_{j}^{k}$ (recall that we have established that these are, respectively, a left and a corresponding right eigenvector associated with $\lambda$ ), then multiplying on the left by the row vector $e_{1}^{T} \in \mathbb{R}^{1 \times n_{j}}$ we obtain

$$
e_{1}^{T}\left(T_{j}^{k}\right)^{T} J=\left(T_{j}^{k} e_{1}\right)^{T} J=e_{1}^{T} \Sigma_{n_{j}} U_{j}^{k}=e_{n_{j}}^{T} U_{j}^{k},
$$

which, read column-wise, leads to

$$
y_{j}^{k}=\left(x_{j}^{k}\right)^{T} J
$$

which, in turn, induces the relationships

$$
Y_{j}=X_{j}^{T} J
$$

between the left and right eigenvector matrices corresponding to blocks of size $n_{j}$ associated with $\lambda$.

If $S \in \mathbb{R}^{2 n \times 2 n}$, we obtain a similar result, but including the signs for the blocks of size $n_{j}$, namely

$$
y_{j}^{k}=\varsigma_{j}^{k}\left(x_{j}^{k}\right)^{T} J, \quad \varsigma_{j}^{k}= \pm 1
$$

which leads to

$$
Y_{j}=\Upsilon_{j} X_{j}^{T} J, \quad \Upsilon_{j}=\operatorname{Diag}\left(\varsigma_{j}^{1}, \cdots, \varsigma_{j}^{r_{j}}\right), \quad X_{j} \in \mathbb{R}^{2 n \times r_{j}} .
$$

- Case 1.b: $\lambda \in\{-1,1\}, n_{j}$ odd

In this case the procedure is similar to the one above: when $n_{j}$ is odd, the blocks in the canonical form are paired as

$$
\left[\frac{U_{j}^{k}}{U_{j}^{k+1}}\right] S\left[T_{j}^{k} \mid T_{j}^{k+1}\right]=\left[\begin{array}{l|l}
\mathcal{J}_{n_{j}}(\lambda) & \\
\hline & \mathcal{J}_{n_{j}}(\lambda)^{-T}
\end{array}\right] .
$$


To transform the structured canonical form into the Jordan form, we can use the similarity matrices $D_{n_{j}}$ satisfying (25) or, equivalently,

$$
\mathcal{J}_{n_{j}}\left(\lambda^{-1}\right)=\left(D_{n_{j}}\right)^{-1} \mathcal{J}_{n_{j}}(\lambda)^{-T} D_{n_{j}},
$$

which can also be written as

$$
\mathcal{J}_{n_{j}}(\lambda)^{T}\left(-D_{n_{j}}\right) \mathcal{J}_{n_{j}}\left(\lambda^{-1}\right)=-D_{n_{j}}
$$

where

$$
D_{n_{j}}=\left[\begin{array}{ccc}
0 & \overrightarrow{0}^{T} & 1 \\
\overrightarrow{0} & * & * \\
1 & * & *
\end{array}\right]
$$

as in $\S 3.2$ above, where $\overrightarrow{0}$ represents the zero vector of $\mathbb{R}^{n_{j}-2}$. Now, $D_{n}$ satisfies

$$
\left[\begin{array}{l|l}
I_{n_{j}} & \\
\hline & \left(-D_{n_{j}}\right)^{-1}
\end{array}\right]\left[\begin{array}{l|l}
\mathcal{J}_{n_{j}}(\lambda) & \\
\hline & \mathcal{J}_{n_{j}}(\lambda)^{-T}
\end{array}\right]\left[\begin{array}{l|l}
I_{n_{j}} & \\
\hline & -D_{n_{j}}
\end{array}\right]=\left[\begin{array}{ll|l}
\mathcal{J}_{n_{j}}(\lambda) & \\
\hline & \mathcal{J}_{n_{j}}(\lambda)
\end{array}\right] .
$$

Replacing this into (33) leads to

$$
\left[\frac{U_{j}^{k}}{\left(-D_{n_{j}}\right)^{-1} U_{j}^{k+1}}\right] S\left[T_{j}^{k} \mid T_{j}^{k+1}\left(-D_{n_{j}}\right)\right]=\left[\begin{array}{l|l}
\mathcal{J}_{n_{j}}(\lambda) & \\
\hline & \mathcal{J}_{n_{j}}(\lambda)
\end{array}\right]
$$

but we know from (6) that

$$
\left[\frac{Q_{j}^{k}}{Q_{j}^{k+1}}\right] S\left[P_{j}^{k} \mid P_{j}^{k+1}\right]=\operatorname{Diag}\left(\mathcal{J}_{n_{j}}, \mathcal{J}_{n_{j}}\right)
$$

so we may identify

$$
\begin{array}{ll}
P_{j}^{k}=T_{j}^{k}, & P_{j}^{k+1}=T_{j}^{k+1}\left(-D_{n_{j}}\right), \\
Q_{j}^{k}=U_{j}^{k}, & Q_{j}^{k+1}=\left(-D_{n_{j}}\right)^{-1} U_{j}^{k+1} .
\end{array}
$$

Now, recall that the blocks $T_{j}^{k}$ and $U_{j}^{k}$ in the symplectic canonical form are linked via

$$
\left[\frac{\left(T_{j}^{k}\right)^{T}}{\left(_{j}^{k+1}\right)^{T}}\right] J=\left[\begin{array}{l|l} 
& I_{n_{j}} \\
\hline-I_{n_{j}} &
\end{array}\right]\left[\begin{array}{c}
U_{j}^{k} \\
\hline U_{j}^{k+1}
\end{array}\right]
$$

or, in short, $\left(T_{j}^{k}\right)^{T} J=U_{j}^{k+1},\left(T_{j}^{k+1}\right)^{T} J=-U_{j}^{k}$. Substituting this into (35), we obtain for every $j \in\{1, \ldots, q\}$ and every $k=1,3,5, \cdots, r_{j}-1$ the identities

$$
\left(P_{j}^{k}\right)^{T} J=-D_{n_{j}} Q_{j}^{k+1}, \quad\left(P_{j}^{k+1}\right)^{T} J=D_{n_{j}} Q_{j}^{k}
$$

between the $P_{j}^{k}$ and $Q_{j}^{k}$ blocks. The special form (34) of $D_{n_{j}}$ implies that $e_{1}^{T} D_{n_{j}}=e_{n_{j}}^{T}$, so if we denote right and left eigenvectors by $x_{j}^{k}$ and $y_{j}^{k}$, respectively, as before, then

$$
\left(x_{j}^{k}\right)^{T} J=-e_{1}^{T} D_{n_{j}} Q_{j}^{k+1}=-y_{j}^{k+1}
$$

and

$$
\left(x_{j}^{k+1}\right)^{T} J=\left(e_{1}^{T} D_{n_{j}}\right) Q_{j}^{k}=y_{j}^{k},
$$


which can be summarized in matrix form as

$$
Y_{j}=\Theta_{j} X_{j}^{T} J, \quad \Theta_{j}=\left(\Sigma_{2} \oplus \cdots \oplus \Sigma_{2}\right) \in \mathbb{R}^{r_{j} \times r_{j}}, \quad X_{j} \in \mathbb{C}^{2 n \times r_{j}} .
$$

Since there is no difference here between the real and complex canonical forms, the only difference in the equality above whenever $S \in \mathbb{R}^{2 n \times 2 n}$ is that $X_{j} \in \mathbb{R}^{2 n \times r_{j}}$.

Finally, we summarize the relationship between the matrices $W_{j}$ and $Z_{j}$ in (9), i.e., between the matrices of left and right eigenvectors for all Jordan blocks associated with 1 or -1 with size at least $n_{j}$ as follows:

$$
W_{j}=\left(\Lambda_{1} \oplus \Lambda_{2} \oplus \cdots \oplus \Lambda_{j}\right) Z_{j}^{T} J
$$

where, for each $k=1, \ldots, j$,

- $\Lambda_{k}=\Theta_{k}$ if $n_{k}$ is odd,

- $\Lambda_{k}=\Upsilon_{k}$ if $n_{k}$ is even and $S \in \mathbb{R}^{2 n \times 2 n}$, and

- $\Lambda_{k}=I_{k}$ if $n_{k}$ is even and $S \in \mathbb{C}^{2 n \times 2 n}$.

\subsubsection{Case 2: $\lambda \in \mathbb{C} \backslash\{-1,1\}$}

This case is treated similarly to the case $\lambda= \pm 1$ with odd $n_{j}$ described in Case 1.b of $\S$ 3.3.1: the transformations leading to Jordan chains from canonical vectors are the same, with the only difference that now $\lambda^{-1} \neq \lambda$, so we have two identical Jordan structures, each one associated with either eigenvalue $\lambda$ or $\lambda^{-1}$. Recall that, for each $j \in\{1, \ldots, q\}$, the $2 n \times r_{j}$ matrix $X_{j}$ (resp., the $r_{j} \times 2 n$ matrix $Y_{j}$ ) collects all right (resp., left) eigenvectors of $S$ corresponding to Jordan blocks of size $n_{j}$ associated with $\lambda$. Let $\widetilde{X}_{j}$ (resp., $\widetilde{Y}_{j}$ ) be the analogous matrix associated with the reciprocal eigenvalue $1 / \lambda$. Then one can prove that

$$
Y_{j}=-\tilde{X}_{j}^{T} J, \quad \tilde{Y}_{j}=\left(\frac{-1}{\lambda^{2}}\right)^{n_{j}-1} X_{j}^{T} J
$$

or, if we lump together the right (resp., left) eigenvector matrices into $Z_{s}, \widetilde{Z}_{s}$ (resp., $W_{s}, \widetilde{W}_{s}$ ), that

$$
W_{s}=-\widetilde{Z}_{s}^{T} J, \quad \widetilde{W}_{s}=\Theta_{s} Z_{s}^{T} J,
$$

where

$$
\Theta_{s}=\left(\left(\frac{-1}{\lambda^{2}}\right)^{n_{1}-1} I_{r_{1}}\right) \oplus \ldots \oplus\left(\left(\frac{-1}{\lambda^{2}}\right)^{n_{s}-1} I_{r_{s}}\right) .
$$

We conclude by observing that if $\lambda \in \mathbb{R}$ and $S \in \mathbb{R}^{2 n \times 2 n}$, then the eigenvectors in the formulas above can all be chosen to be real.

Special Case: $S \in \mathbb{R}^{2 n \times 2 n} \wedge \lambda \in \mathbb{C},|\lambda|=1$.

The relationships we have just obtained when $\lambda \neq \pm 1$ are not direct relationships between left and right eigenvectors associated with $\lambda$, but crossed relationships between left eigenvectors associated with $\lambda$ and right eigenvectors associated with $\lambda^{-1}$ (and viceversa). In the special case when $S$ is real and $\lambda$ lies on the unit circle we can do better, actually finding direct relationships for left and right $\lambda$-eigenvectors: we proceed as before, using the transformations in (30) to obtain the corresponding Jordan chains for the blocks in (24). This leads to the relationships

$$
\left(x_{j}^{k}\right)^{*}(i J)=(\overline{i \lambda})^{n_{j}-1} \varsigma_{j}^{k} y_{j}^{k}
$$


between the left eigenvectors $y_{j}^{k}$ and the right eigenvectors $x_{j}^{k}$ associated with $\lambda$ in the $k$-th Jordan block of size $n_{j}$ (recall that $\varsigma_{j}^{k}$ is the sign of the $k$-th Jordan block of size $n_{j}$ ).

Thus, the relationship between the matrices $W_{s}$ and $Z_{s}$ of left and right eigenvectors in (9) is

$$
\left(Z_{s}\right)^{*}(i J)=\operatorname{Diag}\left\{\Lambda_{1}, \ldots \Lambda_{s}\right\} W_{s},
$$

where

$$
\Lambda_{j}=(\overline{i \lambda})^{n_{j}-1} \operatorname{diag}\left\{\varsigma_{j}^{1}, \ldots, \varsigma_{j}^{r_{j}}\right\}, \quad j=1, \ldots, s .
$$

Note that in this case the upper triangular Toeplitz matrix we need to transform into a Jordan block has $t_{1}=i \lambda$ on the superdiagonal, so in this case the matrix corresponding to the change of basis has the form

$$
A_{n_{j}}=\left[\begin{array}{c|c}
A_{n_{j}-1} & * \\
\hline 0_{1 \times\left(n_{j}-1\right)} & (i \lambda)^{-\left(n_{j}-1\right)}
\end{array}\right] .
$$

We conclude by observing that this result is valid also when $S \in \mathbb{C}^{2 n \times 2 n}$ and $S^{*} J S=J$, where $*$ denotes the conjugate transpose, since in this case the matrix $S$ is also $(i J)$-unitary.

\section{Asymptotic expansions for structured symplectic perturbations}

Once the relations are known between left and right eigenvectors in the Jordan form of $S$, we incorporate that information into the formulas in Theorem 2.3 in order to refine them. These formulas depend, in principle, on the eigenvalues of the Schur complement $\left(\Phi_{j} / \Phi_{j-1}\right)(B)$ in (12), but we shall see that, once the relations between left and right eigenvectors are incorporated, the relevant Schur complements can be written in terms of a slightly different family of nested matrices:

Definition 4.1 Let $\lambda$ be an eigenvalue of a symplectic matrix $S$ with Jordan structure (6), and let $j \in$ $\{1, \ldots, q\}$. Then, for every matrix $K$ with the same dimensions as $S$, we define

$$
\Xi_{j}(K)=Z_{j}^{*} K Z_{j}
$$

where the columns of $Z_{j}$ are right eigenvectors of $S$, associated with Jordan blocks corresponding to $\lambda$ of size at least $n_{j}$, which have been extracted from one of the symplectic canonical forms in $\S 3.1$ as explained in $\S 3.2$.

Notice that this definition imposes a nested structure on the matrices $\Xi_{s}(K)$, just as on the matrices $\Phi_{s}(K)$ before. Hence, for each $s$ we can define the Schur complement of $\Xi_{s-1}(K)$ in $\Xi_{s}(K)$, and denote it by $\left(\Xi_{s} / \Xi_{s-1}\right)(K)$. One can easily check that

$$
\left(\Xi_{s} / \Xi_{s-1}\right)(K)=X_{s}^{*}\left(K-K Z_{s-1} \Xi_{s-1}(K)^{-1} Z_{s-1}^{*} K\right) X_{s},
$$

where $X_{s}$ is the submatrix of $Z_{s}$ containing all eigenvectors associated with Jordan blocks of size exactly $n_{s}$.

As before, we distinguish several cases, depending on whether the unperturbed eigenvalue $\lambda$ belongs to $\{1,-1\}$ or not, and on whether $S$ is real or complex. Each of these cases will be illustrated with one or more numerical examples in Section 5 below. Also for each case we shall specialize the results in the simplest case when the perturbation $\widehat{S}(\varepsilon)-S$ has rank one, not only because it is the situation most commonly studied in the literature, but because the asymptotic formulas come out especially simple, revealing the influence of the sign characteristic on the behavior of perturbed eigenvalues. 


\subsection{Asymptotic expansions: the case $\lambda \notin\{-1,1\}$}

In this case each eigenvalue $\lambda$ is paired with its reciprocal $\lambda^{-1}$, which is also an eigenvalue of $S$ with the same Jordan structure as $\lambda$. The relations (39) between normalized eigenvectors corresponding to $\lambda$ and $\lambda^{-1}$ lead to the connection

$$
\left(\widetilde{\Phi}_{j} / \widetilde{\Phi}_{j-1}\right)(B)=-\left(\frac{-1}{\lambda^{2}}\right)^{n_{j}-1}\left(\Phi_{j} / \Phi_{j-1}\right)(B)^{T}
$$

between the Schur complements associated with $\lambda$ and $1 / \lambda$. This induces a one-to-one relationship between each eigenvalue $\xi$ of $\left(\Phi_{j} / \Phi_{j-1}\right)(B)$ and the corresponding eigenvalue $\widetilde{\xi}=-\left(\frac{-1}{\lambda^{2}}\right)^{n_{j}-1} \xi$ of $\left(\widetilde{\Phi}_{j} / \widetilde{\Phi}_{j-1}\right)(B)$. Replacing these values in formula (13) of Theorem 2.3 does little more than confirming the symplectic symmetry of perturbed eigenvalues (which was known in advance, since $\widehat{S}$ is assumed to be symplectic).

\subsection{Asymptotic expansions: the case $S, \widehat{S} \in \mathbb{R}^{2 n \times 2 n}$ with $|\lambda|=1$}

We address this situation separately, since, as we have seen in $\S 3.3 .2$, more specific information is available in this special case to refine the asymptotic expansions: if $S, \widehat{S} \in \mathbb{R}^{2 n \times 2 n}$ and $|\lambda|=1$, we can make use of the left-right eigenvector relationships (40) obtained in $\S$ 3.3.2. Since these connect left and right eigenvectors both associated with $\lambda$, we may further simplify the Schur complements $\left(\Phi_{j} / \Phi_{j-1}\right)(B)$ : one can easily check that if $\Phi_{j-1}(B)$ is nonsingular, then

$$
\left(\Phi_{j} / \Phi_{j-1}\right)(B)=\mathrm{i}^{n_{j}} \lambda^{n_{j}-1} H_{j},
$$

where $H_{j}=\operatorname{Diag}\left\{\varsigma_{j}^{1}, \ldots, \varsigma_{j}^{r_{j}}\right\}\left(\Xi_{j} / \Xi_{j-1}\right)(J B)$, where the $\varsigma_{j}^{k}$ are the signs associated with $n_{j} \times n_{j}$ Jordan blocks, and $\Xi_{j-1}$ and $\Xi_{j}$ are as in Definition 4.1. This proves the following result, which describes the structured asymptotic expansions obtained from Theorem 2.3 in this special case.

Theorem 4.2 Let $\lambda$ be an eigenvalue of a real symplectic matrix $S$ with $|\lambda|=1$ and Jordan structure (6). Let $\widehat{S}(\varepsilon)=\left(I+\varepsilon B+O\left(\varepsilon^{2}\right)\right) S$ be an arbitrary real symplectic structured perturbation of $S$ as in (3). Let $j \in\{1, \ldots, q\}$ be given and assume that $\Xi_{j-1}(J B)$ is nonsingular if $j>1$, where $\Xi_{j-1}(\cdot)$ is given by Definition 4.1. Then there are $r_{j} n_{j}$ eigenvalues of the perturbed matrix $\widehat{S}(\varepsilon)$ admitting first order expansions

$$
\widehat{\lambda}_{j, k, l}(\varepsilon)=\lambda\left(1+i\left(\xi_{j, k}\right)^{\frac{1}{n_{j}}} \varepsilon^{\frac{1}{n_{j}}}\right)+o\left(\varepsilon^{\frac{1}{n_{j}}}\right)
$$

where $\xi_{j, k}, k=1, \ldots, r_{j}$, is any of the eigenvalues of the matrix

$$
H_{j}=\operatorname{Diag}\left\{\varsigma_{j}^{1}, \ldots, \varsigma_{j}^{r_{j}}\right\}\left(\Xi_{j} / \Xi_{j-1}\right)(J B)
$$

if $j>1$, or of the matrix $H_{1}=\operatorname{Diag}\left\{\varsigma_{1}^{1}, \ldots, \varsigma_{1}^{r_{1}}\right\} \Xi_{1}(J B)$ if $j=1$. The values $\widehat{\lambda}_{j, k, l}(\varepsilon)$ for $l=1, \ldots, n_{j}$ are defined by taking the $n_{j}$-th roots of $\xi_{j, k}$ (those are pairwise distinct unless $\xi_{j, k}=0$ ).

Notice that, since we are in the real case, the matrix $B$ is real Hamiltonian and, consequently, $\left(\Xi_{j} / \Xi_{j-1}\right)(J B)$ is Hermitian. Although $H_{j}$ is a complex matrix and, in fact, may have complex eigenvalues depending on the signs $\varsigma_{j}^{k}$, its characteristic polynomial is always real. Thus, the spectrum of $H_{j}$ is symmetric with respect to the real line, so the perturbed asymptotic expansions are consistent with the real symmetry of the spectrum of $\widehat{S}$.

Now, we analyze the special case when $\xi_{j, k} \in \mathbb{R}$; this happens, for instance, for rank-one perturbations, or whenever all Jordan blocks of size $n_{j}$ associated with $\lambda$ have the same sign in the sign characteristic. In this case, more specific information can be gathered about the behavior of perturbed eigenvalues. We assume $\xi_{j, k} \neq 0$ and, as usual, distinguish two cases:

- $n_{j}$ is odd

In this case, one of the $n_{j}$-th roots of $\xi_{j, k}$ must be real, so one of the perturbed eigenvalues moves tangentially to the unit circle, according to Theorem 4.2. Such a single eigenvalue cannot actually leave the unit circle, since that would break the symplectic spectral symmetry. Hence, one of the perturbed eigenvalues moves on the unit circle, and as to the remaining eigenvalues, half of them move inwards, and the other half outwards of the unit circle. 
- $n_{j}$ is even

In this case we need to take into account not only the sign of $\xi_{j, k}$, but the remainder of the integer division of $n_{j}$ by 4 as well. The following table summarizes all possibilities, depending on the nature of the $n_{j}$-th roots of $\xi_{j, k}$ :

\begin{tabular}{l|l|l}
$\xi_{j, k}>0$ & $\begin{array}{l}n_{j}=4 p \\
\begin{array}{l}\text { Two eigenvalues move on the unit cir- } \\
\text { cle in opposite directions, another two } \\
\text { move away from the unit circle orthog- } \\
\text { onally to it, one inwards and the other } \\
\text { one outwards. The remaining } 4(p-1) \\
\text { eigenvalues move away from the unit } \\
\text { circle, half inwards and half outwards. }\end{array}\end{array}$ & $\begin{array}{l}\text { Two eigenvalues move on the unit cir- } \\
\text { cle in opposite directions, and the re- } \\
\text { maining } 4 p \text { move away from the cir- } \\
\text { cle, half inwards and half outwards, } \\
\text { none of them orthogonally to the circle. }\end{array}$ \\
\hline$\xi_{j, k}<0$ & $\begin{array}{l}2 p \text { eigenvalues move inside the unit } \\
\text { circle and the remaining 2p eigenval- } \\
\text { ues move outside. None of them moves } \\
\text { orthogonally to the circle, none of them } \\
\text { stays on the unit circle. }\end{array}$ & $\begin{array}{l}\text { Two perturbed eigenvalues move away } \\
\text { from the unit circle orthogonally to it, } \\
\text { one inwards and the other one out- } \\
\text { wards. The remaining } 4 p \text { eigenvalues } \\
\text { move away from the unit circle, half in- } \\
\text { wards and half outwards. }\end{array}$
\end{tabular}

Table 4.2

\subsubsection{The case $S, \widehat{S} \in \mathbb{R}^{2 n \times 2 n}$ with $|\lambda|=1$ : Rank one perturbations}

We now specialize the expansions (42) to the case when $\operatorname{rank}(\widehat{S}(\varepsilon)-S)=1$. Notice that this, together with the $J$-Hamiltonian character of $B$, implies that $B J$ is symmetric with rank one. It follows that either $B=u u^{T} J$ for some $u \in \mathbb{C}^{2 n}$ in the complex case, or $B= \pm u u^{T} J$ for some $u \in \mathbb{R}^{2 n}$ in the real case.

Let $\xi=\operatorname{trace}\left(\Xi_{1}(J B)\right)$ be the only nonzero eigenvalue of $H_{1}=\Upsilon_{1} \Xi_{1}(J B)=\Upsilon_{1} X_{1}^{*} J B X_{1}$, where the asterisk denotes the conjugate transpose and $\Upsilon_{1}$ is a $n_{1} \times n_{1}$ diagonal matrix with the signs of the Jordan blocks for $\lambda$ of size $n_{1}$ on its main diagonal. Hence,

$$
\xi=\operatorname{trace}\left(\Xi_{1}(J B)\right)= \pm \operatorname{trace}(\Upsilon_{1} \underbrace{\left(X_{1}^{*} J u\right)}_{a} \underbrace{\left(u^{T} J X_{1}\right)}_{-a^{*}})=\mp \sum_{j=1}^{r_{1}} \varsigma_{j}\left|a_{j}\right|^{2},
$$

where the $\varsigma_{j}$ are the signs on the main diagonal of $\Upsilon_{1}$ and the vector $a$ with entries $a_{i}$ is $a=X_{1}^{*} J u$. The asymptotic expansions for the $n_{1}$ perturbed eigenvalues created by the destruction of one largest Jordan block are

$$
\widehat{\lambda}_{k}(\varepsilon)=\lambda\left(1+i \xi^{\frac{1}{n_{1}}} \varepsilon^{\frac{1}{n_{1}}}\right)+o\left(\varepsilon^{\frac{1}{n_{1}}}\right)
$$

where

$$
\xi=\mp \sum_{j=1}^{r_{1}} \varsigma_{j}\left|a_{j}\right|^{2} \in \mathbb{R} .
$$

Thus, the behaviour of the perturbed eigenvalues depends basically on the sign of $\xi$ and on the remainder modulus 4 of $n_{1}$, as already discussed above in Table 4.2 . 


\subsection{Asymptotic expansions: the case $\lambda \in\{-1,1\}$.}

In this situation we expect quite different behaviors depending on the parity of $n_{j}$, since the formulas in $\S 3.3 .1$ are different depending on whether $n_{j}$ is even or odd. Moreover, recall from Remark 3.2 that if $n_{j}$ is even, there can be any number of $n_{j} \times n_{j}$ blocks in the Jordan form of $S$, whereas if $n_{j}$ is odd, there can only be an even number of blocks of size $n_{j}$. This distinction is important, as we shall soon see.

Since the leading coefficients of the asymptotic expansions are given by the eigenvalues of the Schur complements $\left(\Phi_{j} / \Phi_{j-1}\right)(B)$, we may, as before, use the relationships (32) and (37), replacing them in (12) to define

$$
H_{j}=\left(\Phi_{j} / \Phi_{j-1}\right)(B)=\Lambda_{j}\left(\Xi_{j} / \Xi_{j-1}\right)(J B),
$$

where each matrix $\Lambda_{j}$ is either a diagonal matrix of signs if $n_{j}$ is even, or a block-diagonal matrix with $\frac{r_{j}}{2}$ diagonal blocks $\Sigma_{2}=\left[\begin{array}{rr}0 & 1 \\ -1 & 0\end{array}\right]$ if $n_{j}$ is odd. As in $\S 4.2$ above, we incorporate this information into Theorem 2.3 to obtain the following straightforward consequence of it:

Theorem 4.3 Let $\lambda= \pm 1$ be an eigenvalue of a symplectic matrix $S$ with Jordan structure (6). Let $\widehat{S}(\varepsilon)=$ $\left(I+\varepsilon B+O\left(\varepsilon^{2}\right)\right) S$ be an arbitrary symplectic structured perturbation of $S$ as in (3). Let $j \in\{1, \ldots, q\}$ be given and assume that $\Xi_{j-1}(J B)$ is nonsingular if $j>1$, where $\Xi_{j-1}(\cdot)$ is as in Definition 4.1. Then there are $r_{j} n_{j}$ eigenvalues of the perturbed matrix $\widehat{S}(\varepsilon)$ admitting first order expansions

$$
\widehat{\lambda}_{j, k, l}(\varepsilon)=\lambda\left(1+\xi_{j, k}^{\frac{1}{n_{j}}} \varepsilon^{\frac{1}{n_{j}}}\right)+o\left(\varepsilon^{\frac{1}{n_{j}}}\right), \quad k=0,1, \cdots, n_{j}-1,
$$

where $\xi_{j, k}$ is any of the eigenvalues of the matrix

$$
H_{j}=\Lambda_{j}\left(\Xi_{j} / \Xi_{j-1}\right)(J B)
$$

for $j>1$, or of the matrix $H_{1}=\Lambda_{1} \Xi_{1}(J B)$ if $j=1$. The matrix $\Lambda_{j}$ is either a diagonal of signs if $n_{j}$ is even, or a block-diagonal matrix with $\frac{r_{j}}{2}$ diagonal blocks $\Sigma_{2}=\left[\begin{array}{rr}0 & 1 \\ -1 & 0\end{array}\right]$ if $n_{j}$ is odd. The values $\widehat{\lambda}_{j, k, l}(\varepsilon)$ for $l=1, \ldots, n_{j}$ are defined by taking the $n_{j}$-th roots of $\xi_{j, k}$ (those are pairwise distinct unless $\xi_{j, k}=0$ ).

In order to better describe these expansions, we distinguish two cases, depending on the parity of $n_{j}$ :

\subsection{1 $n_{j}$ is even}

In this case, $H_{j}=\operatorname{Diag}\left\{\varsigma_{j}^{1}, \ldots, \varsigma_{j}^{r_{j}}\right\}\left(\Xi_{j} / \Xi_{j-1}\right)(J B)$, but now the Schur complement $\left(\Xi_{j} / \Xi_{j-1}\right)(J B)$ is not in general Hermitian: it only is if both $S, \widehat{S}$ are real, but this is just a particular case of the one treated in $\S 4.2$. Hence, for complex perturbations, the perturbed eigenvalues may move away from $\lambda$ in any direction, although we observe that the expansions are still consistent with the symplectic spectral symmetry, since $n_{j}$ is even, and hence the $n_{j}$-th roots of $\xi$ in (44) can be split into opposite pairs. If both $S, \widehat{S}$ are real, the perturbed eigenvalues can still move away from $\lambda$ in any direction, since $H_{j}$ may have nonreal eigenvalues. In the special case when $H_{j}$ has some real eigenvalue $\xi_{j, k}$, the behavior of the perturbed eigenvalues is the same described in Table 4.2, i.e., the one corresponding to the asymptotic expansions (44), which are basically the same as the ones in (42) (up to a factor $i$ ).

- The case $\lambda \in\{-1,1\}, n_{1}$ even: Rank one perturbations. Suppose, as before, that the rank of $\widehat{S}-S$ is one. We distinguish between the cases of real and complex matrices:

(a) The real case: $S, \widehat{S} \in \mathbb{R}^{2 n \times 2 n}$

This is a particular case of 4.2.1 and what we obtain is basically the same but for $\lambda= \pm 1$ and a real eigenvector matrix $X_{1}$. Recall that $B= \pm u u^{T} J$ and

$$
H_{1}=\Upsilon_{1} \Xi_{1}(J B)= \pm \Upsilon_{1} X_{1}^{T} J u u^{T} J X_{1}=\mp \Upsilon_{1} a a^{T},
$$


where, $a=X_{1}^{T} J u \in \mathbb{R}^{r_{1}}$. Hence,

$$
\xi=\operatorname{trace}\left(\Phi_{1}(B)\right)=\mp \sum_{j=1}^{r_{1}} \varsigma_{1}^{j}\left(a_{j}\right)^{2} \in \mathbb{R} .
$$

As before, the fact that $n_{1}$ is even reduces the analysis of whether the perturbed eigenvalues stay on the unit circle to a discussion depending on the sign of $\xi$ and the divisibility of $n_{1}$ by 4 , as discussed in $\S$ 4.3.1. The four possible situations are summarized in Table 4.2, by just taking $\xi_{j, k}=i^{n_{1}} \xi$.

We stress that, although the asymptotic expansions (44) give only information about the tangents to the 'escape directions' of the perturbed eigenvalues, and therefore are, in principle, inconclusive as to whether the eigenvalues stay or not on the unit circle, they are actually forced to stay in this case by the symmetry constraints imposed by symplecticity, at least until they meet another eigenvalue with opposite sign in the sign characteristic (see Section 4.4 for more details about this).

(b) The complex case: $S, \widehat{S} \in \mathbb{C}^{2 n \times 2 n}$

In this case there are no signs, since there is no sign characteristic, so $\Upsilon_{1}=I_{r_{1}}$ and

$$
\Phi_{1}(B)=X_{1}^{T} J u u^{T} J X_{1} \in \mathbb{C}^{r_{1} \times r_{1}},
$$

so

$$
\xi=-\operatorname{trace}\left(a a^{T}\right)=-\sum_{i=1}^{r_{1}} a_{i}^{2} \in \mathbb{C} .
$$

In the case that, by chance, $\xi$ happens to be real, then (again depending on the parity of $n$ and the sign of $\xi$ ) there may be two perturbed eigenvalues whose escape direction is tangential to the unit circle and that move away in opposite directions. However, in contrast to case (a) these eigenvalues need not stay on the unit circle, because in this case no structural constraints require them to do so.

\subsection{2 $n_{j}$ is odd}

In this case, the left-right eigenvector relationships (38) imply that the matrix $H_{j}$ in the statement of Theorem 4.3 is

$$
H_{j}=D_{j}\left(\Xi_{j} / \Xi_{j-1}\right)(J B), \quad j>1,
$$

where $D_{j}=\overbrace{\Sigma_{2} \oplus \ldots \oplus \Sigma_{2}}^{\frac{r_{j}}{2} \text { times }}$ (for $j=1$, this formula reduces to $H_{1}=D_{1} \Xi_{1}(J B)$ ). One can easily check that $D_{j}^{-1}=D_{j}^{T}=-D_{j}$, and that $H_{j}$ is $D_{j}$-Hamiltonian. Therefore, the spectrum of $H_{j}$ is symmetric with respect to the origin, and its nonzero eigenvalues can be grouped into pairs $(\xi,-\xi)$, each giving rise to two perturbed eigenvalues with expansions

$$
\begin{aligned}
& \widehat{\lambda}_{k}(\varepsilon)=\lambda\left(1+\xi^{\frac{1}{n_{j}}} \varepsilon^{\frac{1}{n_{j}}}\right)+o\left(\varepsilon^{\frac{1}{n_{j}}}\right), \\
& \widehat{\mu}_{k}(\varepsilon)=\lambda\left(1-\xi^{\frac{1}{n_{j}}} \varepsilon^{\frac{1}{n_{j}}}\right)+o\left(\varepsilon^{\frac{1}{n_{j}}}\right),
\end{aligned}
$$

which reflects the symplectic symmetry of the spectrum of the perturbed matrix $\widehat{S}(\varepsilon)$.

We will now show that there are situations when the Schur complement must have at least one zero eigenvalue depending on the case whether $\rho$ is odd or even. These situations fall, of course, out of the scope of Theorem 4.3, which only gives relevant information if $\xi_{j, k} \neq 0$

\section{- Case 1: $\rho$ is odd}

We have seen in Remark 2.5 that if $r=\operatorname{rank}(B)$ is given by (14), then the Schur complement $H_{j}=$ $\left(\Phi_{j} / \Phi_{j-1}\right)(B)$ may have at most $\rho$ nonzero eigenvalues. Thus, Theorem 4.3 accounts for at most $\rho n_{j}$ among the perturbed eigenvalues, which will come from $\rho$ destroyed $n_{j} \times n_{j}$ Jordan blocks of $S$. 
Since the nonzero eigenvalues of $H_{j}$ show up in pairs $(\xi,-\xi)$ and the trace of $H_{j}$ is zero, we conclude that in the case that $\rho$ is odd, at least one eigenvalue of $H_{j}$ must be zero. This zero eigenvalue corresponds to $n_{j}$ eigenvalues of $\widehat{S}$ unaccounted for by Theorem 4.3. Of course, the $(\rho-1) n_{j}$ perturbed eigenvalues corresponding to the $\rho-1$ generically nonzero eigenvalues of $H_{j}$ are still given by Theorem 4.3.

This very particular situation, i.e., when both $n_{j}$ and $\rho$ are odd, requires a separate analysis on its own to describe what happens to the 'atypical' $n_{j}$ perturbed eigenvalues corresponding to that zero eigenvalue of $H_{j}$. This can be done by combining results in $[15,1]$ with Newton polygon techniques. Since the proof has to be built from scratch, and requires techniques quite different to the ones employed here, this analysis shall be deferred to a later stage.

\section{- Case 2: $\rho$ is even}

This situation is easier to analyze: the asymptotic expansions for all perturbed eigenvalues with nonzero $\xi$ are those described in (46) (in this case, as in any other one, there may be zero eigenvalues in the Schur complement, corresponding to nongeneric perturbations. The difference with Case $\mathbf{1}$ above is that there the zero eigenvalue must be present).

Notice that both asymptotic expansions in (42) and (46) are essentially the same, up to a factor $i$. The main difference is that now we have a more detailed formula for $H_{j}$. It is straightforward to check that also in this case the spectrum of $H_{j}$ may contain arbitrary complex eigenvalues, so the perturbed eigenvalues can move away from $\lambda$ in any direction. Finally, if $S, \widehat{S} \in \mathbb{R}^{2 n \times 2 n}$ and if $\xi$ is neither real not purely imaginary, then the number of Jordan blocks of size $n_{j}$ destroyed by the perturbation is at least 4 , because the quadruple $\{\xi,-\xi, \bar{\xi},-\bar{\xi}\}$ is in the spectrum of $H_{j}$.

- The case $\lambda \in\{-1,1\}, n_{1}$ odd: Rank one perturbations. The case of odd-sized largest Jordan block and $\operatorname{rank}(B)=1$ constitutes, as already shown, a highly non-generic situation, since one can prove that all the eigenvalues of $\Phi_{1}(B)$ are zero. Thus, the first order perturbation theory we are using provides no information whatsoever on the leading terms, and a completely different analysis has to be performed.

\subsection{Observations on meeting eigenvalues}

The so far obtained results from Section 4 can be used to explain the behavior of eigenvalues of real symplectic matrices under parameter-dependent rank-one perturbations with focus on eigenvalues on the unit circle: let $J \in \mathbb{R}^{2 n \times 2 n}$ be skew-symmetric and invertible, let $S \in \mathbb{R}^{2 n \times 2 n}$ be $J$-symplectic and consider a structurepreserving rank-one perturbation of the form

$$
S(\tau)=\left(I+\tau u u^{T} J\right) S
$$

for some $u \in \mathbb{R}^{2 n \times 2 n}$. Then for each eigenvalue $\lambda_{0}$ of $S=S(0)$, there is an eigenvalue $\lambda(\tau)$ of $S(\tau)$ such that $\lambda(\tau)$ is continuous as a function of $\tau$ and such that $\lambda(0)=\lambda_{0}$.

In the following, we will investigate what happens if two simple eigenvalues meet at 1 for some value $\tau_{0}$ to form a Jordan block of size $2 \times 2$. At first, this may sound like a highly nongeneric situation and one may think that it will almost never be observed in practice. Indeed, on the one hand it was observed in [18] that the eigenvalue curves $\lambda(\tau)$ generically do not intersect, if $S$ is a general complex matrix, and on the other hand the point 1 is a very special point in the complex plane, so even if the curves intersect, why should this be in the special point 1. In our particular situation, however, the matrix $S$ is both real and symplectic. Thus, if $S(\tau)$ has a simple eigenvalue $\lambda(\tau)$ on the unit circle for some $\tau$, then for symmetry reasons it has to stay on the unit circle unlees it meets another eigenvalue on the unit circle, so an intersection of eigenvalue curves for eigenvalues on the unit circle can be expected and this is indeed what can be observed in numerical experiments. Since furthermore $S(\tau)$ is real and thus all its eigenvalues are symmetric with respect to the real line, the values \pm 1 are natural values where eigenvalues moving along the unit circle can (and do) meet.

If we let $\widetilde{S}=S\left(\tau_{0}\right)$ then we can use the results from Table 4.2 to understand what happens at and around the moment $\tau_{0}$ when the two eigenvalues meet and form a Jordan block associated with the eigenvalue one. 
For $\varepsilon \geq 0$ let us define

$$
\begin{aligned}
& S_{f}(\varepsilon):=\left(I+\varepsilon u u^{T} J\right) \widetilde{S}=\left(I+\left(\tau_{0}+\varepsilon\right) u u^{T} J\right) S, \quad \text { and } \\
& S_{p}(\varepsilon):=\left(I+\varepsilon u u^{T}(-J)\right) \widetilde{S}=\left(I+\left(\tau_{0}-\varepsilon\right) u u^{T} J\right) S .
\end{aligned}
$$

Thus, the asymptotic expansion for $S_{f}(\varepsilon)$ will tell us what happens "in the future" when the $2 \times 2$ Jordan block at $\lambda_{0}=1$ will split again, while the asymptotic expansion for $S_{p}(\varepsilon)$ gives information about "the past", i.e., for the time before the two eigenvalues have met and formed the Jordan block. Observe that if $\xi$ is the unique nonzero eigenvalue of $\Phi_{1}\left(u u^{T} J\right)$, then $-\xi$ is the nonzero eigenvalue of $\Phi_{1}\left(u u^{T}(-J)\right)$. Thus, according to Table 4.2, we have the following two situations:

1. If $\xi>0$, then there exists a neighborhood $U$ of $t_{0}$ such that for all $t \in U$ we have:

(a) for increasing $t, t<t_{0}$ the matrix $S(t)$ has two conjugate complex eigenvalues on the unit circle close to $\lambda_{0}=1$ that both move towards $\lambda_{0}=1$;

(b) for increasing $t, t>t_{0}$ the matrix $S(t)$ has two real eigenvalues close to $\lambda_{0}=1$ that are reciprocals of each other and that both move away from $\lambda_{0}=1$.

2. If $\xi<0$, then there exists a neighborhood $U$ of $t_{0}$ such that for all $t \in U$ we have:

(a) for increasing $t, t<t_{0}$ the matrix $S(t)$ has two real eigenvalues close to $\lambda_{0}=1$ that are reciprocals to each other and that both move towards $\lambda_{0}=1$;

(b) for increasing $t, t>t_{0}$ the matrix $S(t)$ has two conjugate complex eigenvalues on the unit circle close to $\lambda_{0}=1$ that both move away from $\lambda_{0}=1$.

\section{Numerical examples and the rank of $B$.}

In this section we illustrate with numerical examples the different expansions derived in Section 4. As noted in Remark 2.6, given any structure-preserving perturbation $\widehat{S}$ of the form (3), there are infinitely many other symplectic perturbations with the same first-order coefficient matrix $B$. Part of the eigenvalues of these matrices (those covered by Theorem 2.3 and its offspring) will have the same leading terms as the ones from $\widehat{S}$, but other eigenvalues may behave differently, either due to the $O\left(\varepsilon^{2}\right)$ coefficient matrix, or even because the (common) Schur complements $H_{j}$ have some zero eigenvalue.

In order to distinctly isolate the behavior of the eigenvalues covered by our analysis, we will associate to each perturbed matrix $\widehat{S}(\varepsilon)$ another symplectic matrix $\widetilde{S}(\varepsilon)$, also of the form (3) and with the same leading matrix $B$, but $\widetilde{S}(\varepsilon)$ will only display those perturbed eigenvalues covered by the theory in Section 4 . The reason for this is that $\widetilde{S}$ will be constructed in such a way that $\operatorname{rank}(B)=\operatorname{rank}(\widetilde{S}-S)$, an identity which is not true, in general, for every structure-preserving perturbation of the form (3). To be more precise, $\widetilde{S}$ will be just the product of exactly $\operatorname{rank}(B)$ rank-one multiplicative $J$-Hamiltonian perturbations (see Appendix A below for the details on this construction). This ensures that each successive rank-one multiplicative perturbation destroys just one single Jordan block, and the corresponding perturbed eigenvalues of $\widetilde{S}$ are described by the expansions in $\S 4$.

Thus, for each situation described in Section 4 we shall specify an unperturbed symplectic matrix with the appropriate spectral structure, and then generate thousands of randomly generated pairs of structure-preserving perturbations $\widehat{S}(\varepsilon)$ and $\widetilde{S}(\varepsilon)$, on which MATLAB R2016B will be run to compute (and plot) their eigenvalues. To be more precise, once the appropriate unperturbed matrix $S$ is chosen,

1. we specify a (low) rank $r$, and generate $r$ random linearly independent ${ }^{3}$ vectors $u_{1}, \ldots, u_{r}$, and a random list of signs $\left\{s_{1}, \ldots, s_{r}\right\}$ to construct a perturbation

$$
\widetilde{S}(\varepsilon)=\left(\prod_{k=1}^{r}\left(I+\varepsilon s_{k} u_{k} u_{k}^{T} J\right)\right) S=(I+\varepsilon B+\cdots) S
$$

as in (47).

\footnotetext{
${ }^{3}$ In order to reduce the workload of the generating process we relax the orthogonality condition on the $u_{j}$ to just linear independence. This is enough to guarantee both rank and symplecticity
} 
2. Once $\widetilde{S}(\varepsilon)$ is constructed, the $2 n \times 2 n$ matrix $B$ above is partitioned into four $n \times n$ blocks, which are then modified according to Lemma 2.1, i.e., if

$$
B=\left[\begin{array}{cc}
B_{11} & B_{12} \\
B_{21} & -B_{11}^{T}
\end{array}\right], \quad B_{12}^{T}=B_{12}, \quad B_{21}^{T}=B_{21},
$$

random $n \times n$ matrices $C_{11}, C_{12}, C_{21}$ are generated, with $C_{12}$ and $C_{21}$ symmetric, in order to construct a perturbation

$$
\widehat{S}(\varepsilon)=\left[\begin{array}{cc}
G & G E \\
C G & G^{-T}+C G E
\end{array}\right] S,
$$

where $G=I_{n}+\varepsilon B_{11}+\varepsilon^{2} C_{11}, \quad E=\varepsilon B_{12}+\varepsilon^{2} C_{12}, \quad C=\varepsilon B_{21}+\varepsilon^{2} C_{21}$. This perturbation will have the same first-order matrix $B$, but the difference $\widehat{S}(\varepsilon)-S$ will have typically full rank.

3. Finally, hundreds of equally spaced values for $\varepsilon$ are sampled from a certain interval $\left[\varepsilon_{\min }, \varepsilon_{\max }\right]$, and the eigenvalues of the corresponding evaluations for each perturbation $\widetilde{S}(\varepsilon)$ and $\widehat{S}(\varepsilon)$ are computed and plotted via MATLAB. Those of $\widetilde{S}(\varepsilon)$ are placed on the left half of the figure, those of $\widehat{S}(\varepsilon)$ on the right half. Perturbed eigenvalues are plotted in red, unperturbed ones in blue.

Example 5.1 The case $S, \widehat{S} \in \mathbb{R}^{2 n \times 2 n},|\lambda|=1$. Let

$$
S=\left[\begin{array}{c|c}
\Lambda & 0 \\
\hline 0 & \Lambda^{-T}
\end{array}\right], \quad \text { for } \quad \Lambda=I_{4} \otimes\left[\begin{array}{rr}
a & b \\
-b & a
\end{array}\right]+J_{8}(0)
$$

with $a^{2}+b^{2}=1$ and $b \neq 0 . S$ is symplectic with four Jordan blocks of size 4, two of them associated with each of the two distinct eigenvalues $\{\lambda, \bar{\lambda}\}$, where $\lambda=a+i b$ lies on the unit circle. Figure 2 displays the eigenvalues of symplectic perturbations to $S$ when $a=\cos (\pi / 40)$ and $b=\sin (\pi / 40)$. The plot in Figure $2 a$ corresponds to perturbations with rank $r=1$, while the one in Figure $2 b$ corresponds to rank $r=2$. In either case, 100 different random structured perturbations $\widetilde{S}(\varepsilon)$ have been created by randomly generating appropriate sets of vectors as described in Step 1 above. Then, each of those 100 perturbations is modified to create the corresponding $\widehat{S}(\varepsilon)$, as described in Step 2. Finally, for each of pair of perturbations $\widetilde{S}(\varepsilon), \widehat{S}(\varepsilon)$, the interval $\left[10^{-6}, 10^{-5}\right]$ for $\varepsilon$ is uniformly sampled with step $10^{-7}$, and MATLAB computes and plots the eigenvalues of the evaluations of both $\widetilde{S}(\varepsilon)$ and $\widehat{S}(\varepsilon)$ at the values of $\varepsilon$ given by the samples.
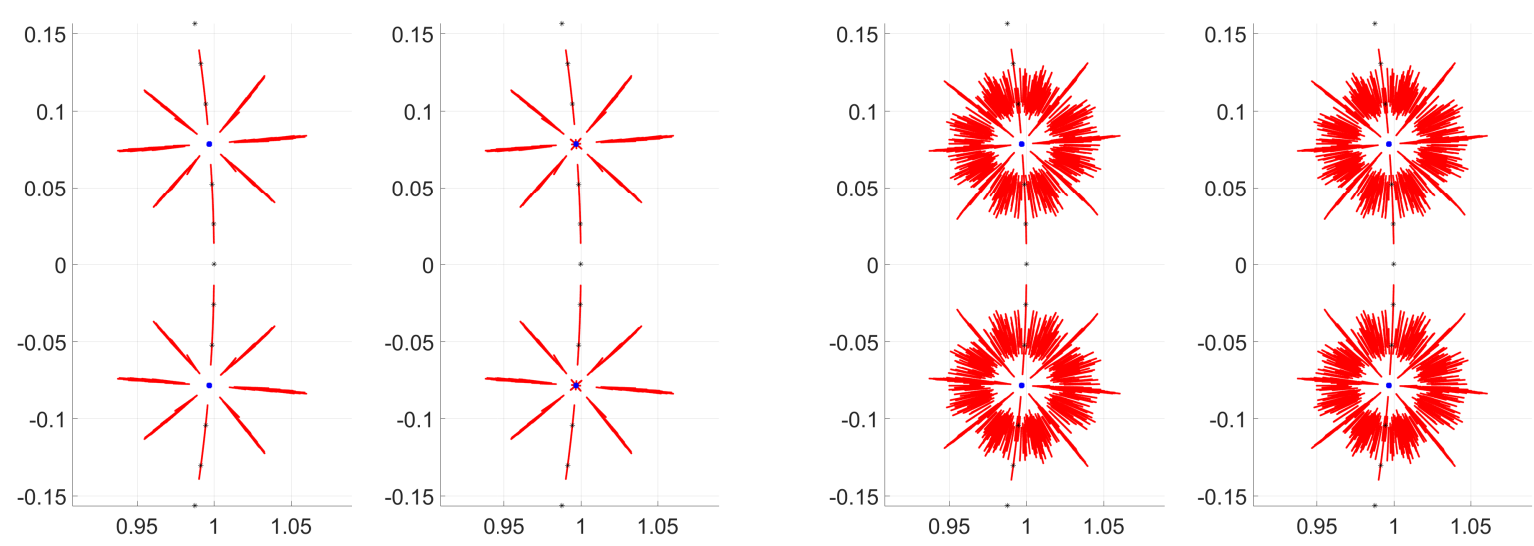

(a) $\operatorname{rank}(B)=1$

(b) $\operatorname{rank}(B)=2$

Figure 2: Low rank perturbations of a symplectic matrix $S$ with two e-vals. on the unit circle

First, we observe that the difference in behaviour for the two ranks is consistent with the expansions in Section 4.2: take first Figure 2a, where $\operatorname{rank}(B)=1$. Since we are in the real case, the number $\xi=\xi_{1, k}$ in 
the asymptotic expansions (42) is a single real number. The 8-legged star visible at each unperturbed eigenvalue on Figure 2a corresponds to the superposition of two rotated versions of the four-legged star depicted by the fourth roots of unity, one for each of the two possible signs for $\xi$ : if $\xi>0$, then two of the four perturbed eigenvalues stay on the unit circle, while the other two move away from it orthogonally, as predicted in Table 4.2. When $\xi<0$, the fourth roots of $\xi$ give rise to the four remaining escape directions in the figure. As to the plot on Figure $2 \mathrm{~b}$, where $\operatorname{rank}(B)=2$, both pairs of Jordan blocks of $S$ associated with $\lambda$ may have opposite signs in the sign characteristic, so the matrix $\Phi_{1}(B)$ may have nonreal eigenvalues. Thus, its fourth roots may be anywhere and, as shown in the plot, the spectra of both $\widetilde{S}$ and $\widehat{S}$ can move away from $\lambda$ in any direction.

Notice that in Figure $2 \mathrm{~b}$, the rank of $B$ is enough for $\widetilde{S}$ to break the two Jordan blocks associated with each eigenvalue. Hence, the asymptotic expansions (42) explain the behaviour of all perturbed eigenvalues, and no significant differences between the spectra of $\widetilde{S}$ and $\widehat{S}$ can be appreciated with the naked eye. In Figure 2a, however, one can spot a very small additional 8-legged star in the graph on the right, corresponding to perturbations of type $\widehat{S}$, which is not present in the one at the left for the auxiliary perturbations $\widetilde{S}$. The reason for this is that $\operatorname{rank}(B)=1$ is not enough to destroy the whole Jordan structure associated with each eigenvalue. The perturbations $\widetilde{S}$ destroy only one Jordan block, while $\widehat{S}$ is in general of full rank and is thus able to undo the complete $\lambda$-Jordan structure of $S$. However, the behaviour of those perturbed eigenvalues which escape fastest away from the unperturbed ones is still the same for both perturbations.

Remark 5.2 The discussion above also explains the different behavior of the two matrices $S_{1}$ and $S_{2}$ in (2) observed in Figure 1. A calculation of the symplectic canonical forms of $S_{1}$ and $S_{2}$, respectively, reveals that the two Jordan blocks of size $n_{1}=1$ associated with the eigenvalue $\lambda=i$ have opposite signs \pm 1 in the sign characteristic for $S_{1}$, but equal signs for $S_{2}$. An analogous observation holds for the eigenvalue $\lambda=-i$. Thus, if we consider random symplectic perturbations of $S_{1}$, then the matrix $\operatorname{Diag}\{+1,-1\} H_{1}$ will generically be nonsymmetric and may thus have complex eigenvalues, so the perturbed eigenvalues may escape into any direction. For $S_{2}$, in contrast, we are exactly in the situation highlighted above, where all signs in the sign characteristic corresponding to Jordan blocks of size $n_{1}$ are equal, resulting in the fact that the eigenvalues $\xi_{k, l}$ are real. Since $n_{1}=1$ is odd, the perturbed eigenvalues coming from each of the two Jordan blocks associated with $\lambda=i$ (or $\lambda=-i$, respectively) have to stay on the unit circle.

Example 5.3 The case $\lambda \in\{-1,1\}, n_{j}$ even. Let

$$
S=\left[\begin{array}{c|c}
\Lambda & 0 \\
\hline 0 & \Lambda^{-T}
\end{array}\right], \quad \text { for } \quad \Lambda=\mathcal{J}_{8}(1) \oplus \mathcal{J}_{8}(1),
$$

where $\mathcal{J}_{8}(1)$ stands for a Jordan block of size 8 associated with $\lambda=1$. The matrix $S$ is symplectic with four Jordan blocks of size 8. As before, Figure 3 displays the eigenvalues of 100 random structured symplectic perturbations of $S$ of the form (3), with $\operatorname{rank}(B)=1$ in Figure $3 a$ and with $\operatorname{rank}(B)=4$ in Figure $3 b$. Within each of the $(a),(b)$ versions, the perturbations of type $\widehat{S}$ are displayed on the left, and those of type $\widehat{S}$ on the right. On the top of each figure we single out one amongst the 100 randomly generated perturbations, at the bottom we show the superposition of the 99 remaining ones. The values for $\varepsilon$ have been sampled from the interval $\left[0,10^{-5}\right]$ at uniform steps of length $10^{-7}$.

The discussion here is more or less the same as in Example 5.1. In fact, for even-sized blocks associated with \pm 1 , the behavior of perturbed eigenvalues is similar to that of those coming from eigenvalues elsewhere on the unit circle, since the asymptotic expansions (44), though more detailed, are basically the same as those in (42).

The main differences between the auxiliary perturbations $\widetilde{S}$ and the standard ones $\widehat{S}$ can be seen, as in Example 5.1 above, only when the rank of $B$ is not enough by itself to destroy the whole Jordan structure associated with $\lambda$ : in Figure 3a, where $\operatorname{rank}(B)=1$, the perturbations $\widetilde{S}(\varepsilon)$ can only break one single $8 \times 8$ Jordan block associated with $\lambda= \pm 1$, while in general, every perturbation $\widehat{S}(\varepsilon)$ destroys the whole $\lambda$-Jordan 
structure. Hence, there is again an additional cluster of eigenvalues for $\widehat{S}(\varepsilon)$ very close to $\lambda=1$. Since these eigenvalues come from the $O\left(\varepsilon^{2}\right)$ terms in $\widehat{S}(\varepsilon)$, their leading exponent is larger than $1 / 8$ and, therefore, they move away from $\lambda$ much slower than the ones coming from the first order term $B$. These coincide, to first order, with the eigenvalues of $\widetilde{S}$.

In Figure $3 \mathrm{~b}$, where $\operatorname{rank}(B)=4$, the first order terms have enough rank to break all Jordan blocks corresponding to $\lambda=1$. Thus, all perturbed eigenvalues are described by Theorem 4.3, and no differences are visible between the left and right plots.
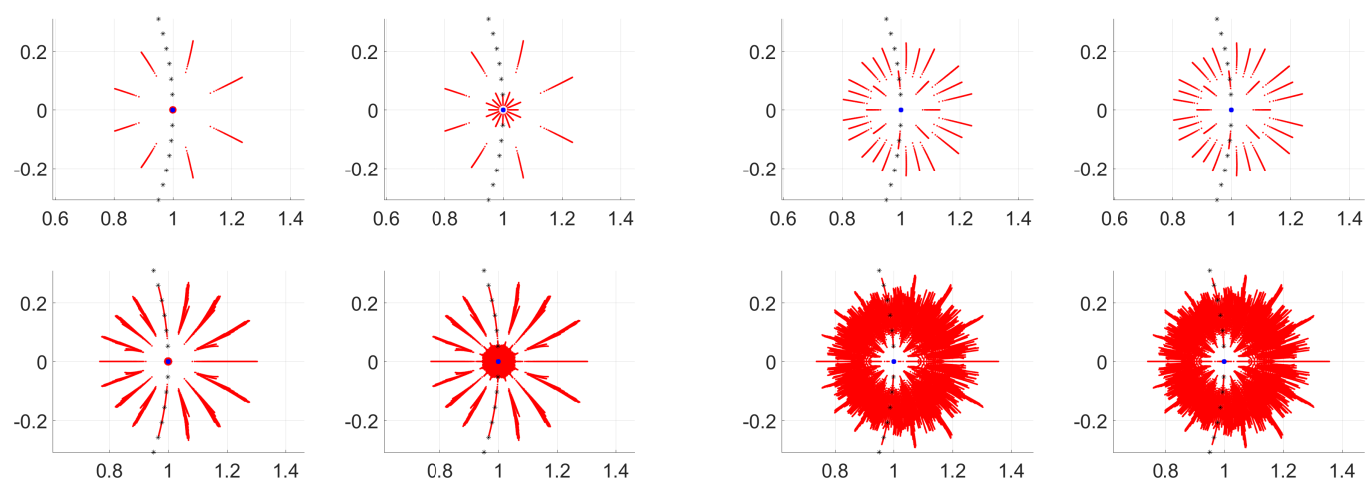

(a) $r=1$

(b) $r=4$

Figure 3:

Example 5.4 . The case $\lambda \in\{-1,1\}, n_{j}$ odd. Let $S$ be a $20 \times 20$ symplectic matrix with the same block structure as the one in Example 5.3, but now taking

$$
\Lambda=\mathcal{J}_{5}(1) \oplus \mathcal{J}_{5}(1) .
$$

Then $S$ has a single eigenvalue $\lambda=1$ with four Jordan blocks of size 5. Notice that, since all blocks are of the same size, $\operatorname{rank}(B)=\rho$, where $\rho$ is given by (14). Hence, $\rho$ is even whenever $\operatorname{rank}(B)$ is either 2 or 4 (any rank larger than or equal to 4 destroys all four Jordan blocks). Figure 4 displays the eigenvalues of 100 random symplectic perturbations of $S$ with the same format as in Figure 3 (i.e., one perturbation singled out at the top, the remaining 99 at the bottom, the auxiliary perturbations $\widetilde{S}$ on the left, and $\widehat{S}$ on the right). Now the values for $\varepsilon$ are taken from partitioning the interval $\left[0,10^{-7}\right]$ with step $10^{-9}$.
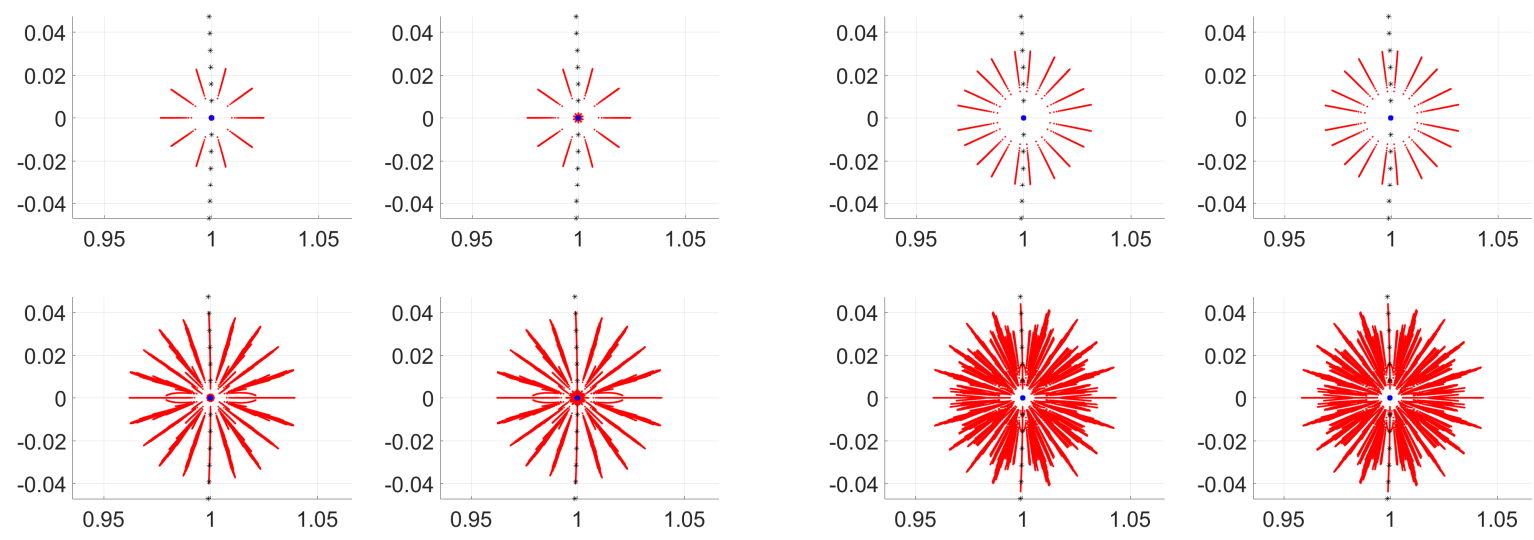

(a) $r=2$

(b) $r=4$

Figure 4:

We only discuss the case when $\rho$ is even (the case when $\rho$ is odd has been left out of our analysis): Figure $4 \mathrm{a}$ corresponds to $\operatorname{rank}(B)=2$ and Figure $4 \mathrm{~b}$ to $\operatorname{rank}(B)=4$. As in the two previous examples, 
no significant differences are visible in Figure $4 \mathrm{~b}$, since the perturbation $\widetilde{S}$ has enough rank to break all Jordan blocks. In Figure 4a, on the other hand, we see on top the superposition of two 5-legged stars, one corresponding to the fifth roots of $\xi$, the other to the fifth roots of $-\xi$, as explained in (46). As in the previous examples, a tiny additional cluster of eigenvalues is visible on the right of Figure 4a for the perturbation $\widehat{S}$, due to the additional rank provided by the $O\left(\varepsilon^{2}\right)$ terms.

\section{References}

[1] Leonhard Batzke, Christian Mehl, André C.M. Ran and Leiba Rodman, Generic rank-k perturbations of structured matrices, Oper. Theory Adv. Appl., Vol. 255, 2016, pp. 27-48.

[2] H. BaumgäRTEL, Analytic Perturbation Theory for Matrices and Operators, Birkhäuser, Basel, 1985.

[3] P. BenneR AND H. FASSBENDER, The symplectic eigenvalue problem, the butterfly form, the SR algorithm, and the Lanczos method, Linear Algebra Appl. Vol. 275-276, 1998, pp. 19-47.

[4] P. BENNER AND H. FASSBENDER, An implicitly restarted symplectic Lanczos method for the symplectic eigevalue problem, SIAM J. Matrix Anal. Appl., Vol. 22 no. 3, 2000, pp. 682-713.

[5] A. Bunse-Gerstner And V. Mehrmann, A symplectic QR-like algorithm for the solution of the real algebraic Riccati equation, IEEE Trans. Automat. Control, Vol. AC-31, 1986, pp. 1104-1113.

[6] F. M. Dopico AND C. R. Johnson, Parametrization of the matrix symplectic group and applications, SIAM J. Matrix Anal. Appl., Vol. 31 no. 2, 2009, pp. 650-673.

[7] H. FAssbender, Symplectic Methods for the Symplectic Eigenvalue Problem, Kluwer Academic/Plenum Publishers, New York, 2000.

[8] S.K. Godunov And M. SAdKane, Spectral Analysis of Symplectic Matrices with Application to the Theory of Parametric Resonance, SIAM J. Matrix Anal. Appl., Vol. 28 no. 4, 2006, pp. 1083-1096.

[9] R. Horn And C. R. Johnson, Matrix Analysis, Cambridge University Press, Cambridge, 1990.

[10] T. KaTO, Perturbation Theory for Linear Operators, Springer, Berlin, 1980.

[11] W.-W. LIN, A new method for computing the closed loop eigenvalues of a discrete-time algebraic Riccati equation, Linear Algebra Appl., Vol. 6, 1987, pp. 157-180.

[12] A.N. Malyshev, Stability radii of symplectic and Hamiltonian matrices, Report No. 160, Department of Informatics, University of Bergen, Norway, 1998.

[13] C. MeHL, On classification of normal matrices in indefinite inner product spaces, Electron. J. Linear Algebra., Vol. 15 , 2006, pp. 50-83.

[14] C. MenL, Essential decomposition of polynomially normal matrices in real indefinite inner product spaces, Electron. J. Linear Algebra., Vol. 15 , 2006, pp. 84-106.

[15] C. Mehl, V. Mehrmann, A. C. Ran And L. Rodman, Eigenvalue perturbation theory of structured matrices under generic structured rank one perturbations: Symplectic, orthogonal, and unitary matrices, BIT Numer. Math., Vol. 54, 2014, pp. 219-255.

[16] V. Mehrmann, A symplectic orthogonal method for single input or single output discrete time optimal linear quadratic control problems, SIAM J. Matrix Anal. Appl., Vol. 9, 1988, pp. 221-248.

[17] J. Moro, J. Burke And M. Overton, On the Lidskii-Vishik-Lyusternik Perturbation theory for eigenvalues of matrices with arbitrary Jordan structure., SIAM J. Matrix Anal. Appl., Vol. 18 no. 4, 1997, pp. 793-817.

[18] A.C.M. RAN AND M. WoJTYŁAK, Eigenvalues of rank one perturbations of unstructured matrices, Linear Algebra Appl., Vol. 437, 2012, pp. 589-600. 
[19] S. V. SAVCHENKO, On the typical change of the spectral properties under a rank-one perturbation, Mat. Zametki, 74 (2003), pp. 590-602 (in Russian).

[20] F. Sos A \& J. Moro, First order asymptotic expansions for eigenvalues of multiplicatively perturbed matrices, SIAM J. Matrix Anal. Appl., Vol. 37 no. 4, 2016, pp. 1478-1504.

\section{A An auxiliary perturbation matrix}

As announced in $\S 5$, we will show that, given any structure-preserving perturbation $\widehat{S}(\varepsilon)$ as defined in (3), one can find structure-preserving perturbations $\widetilde{S}$, also of the form (3) with the same first-order term $B$, but which can be written as a sequence

$$
\widetilde{S}=\widetilde{S}(\varepsilon)=\left(\prod_{k=1}^{r}\left(I+\varepsilon s_{k} u_{k} u_{k}^{T} J\right)\right) S
$$

of $r=\operatorname{rank}(B)$ symplectic multiplicative perturbations, each of whose factors is a rank-one $J$-Hamiltonian perturbation of identity (the $u_{k}$ are vectors, the $s_{k}$ are signs, see Corollary A.2 below). As a consequence of this, the ranks of $\widetilde{S}(\varepsilon)-S$ and $B$ will coincide, and all perturbed eigenvalues of $\widetilde{S}(\varepsilon)$ will be generically covered by the asymptotic expansions derived in Section 4. Those eigenvalues, of course, will have the same first-order behavior as the corresponding eigenvalues of $\widehat{S}(\varepsilon)$, as explained in Remark 2.6.

We begin by observing that $B$ is $J$-Hamiltonian if and only if the matrix $H=-B J$ is complex symmetric or, equivalently, $B=H J$, for some complex symmetric $H$. First, we describe the set of all $2 n \times 2 n$ complex symmetric matrices of given rank $r$ :

Lemma A.1 Let $r \in\{1, \ldots, 2 n\}$. Then

$$
\left\{H \in \mathbb{C}^{2 n \times 2 n}: H^{T}=H, \operatorname{rank}(H)=r\right\}=\left\{\sum_{k=1}^{r} s_{k} u_{k} u_{k}^{T}:\left\{u_{1}, \ldots, u_{r}\right\} \in \mathcal{V}_{r}^{2 n}, s_{k}= \pm 1\right\},
$$

where $\mathcal{V}_{r}^{2 n}$ denotes the family of all orthogonal subsets of $r$ nonzero vectors in $\mathbb{C}^{2 n}$. If $H \in \mathbb{R}^{2 n \times 2 n}$, then $\left\{u_{1}, \ldots, u_{r}\right\}$ can also be chosen to be real.

Proof: Takagi's factorization (see, e.g., Corollary 4.4.4 in [9]) allows us to factor any complex symmetric matrix $H \in \mathbb{C}^{2 n \times 2 n}$ as

$$
H=U D U^{T},
$$

where $U \in \mathbb{C}^{2 n \times 2 n}$ is unitary and $D$ is diagonal with real non-negative diagonal entries. If we denote by $d_{k}, k=1, \ldots, r$, the nonzero entries of $D$ and by $\widetilde{u}_{k}$ the $k$-th column of $U$, then

$$
H=U D U^{T}=\sum_{k=1}^{r} d_{k} \widetilde{u}_{k} \widetilde{u}_{k}^{T}=\sum_{k=1}^{r} s_{k} u_{k} u_{k}^{T}
$$

where

$$
u_{k}=i^{\frac{1-s_{k}}{2}} \sqrt{d_{k}} \widetilde{u}_{k} .
$$

If the matrix $H$ is complex the signs $s_{k}= \pm 1$ can be chosen arbitrarily. If $H$ is real, however, then $H$ is both real and symmetric and, therefore, orthogonally diagonalizable. Hence, $H=W \Lambda W^{T}$ for some orthogonal matrix $W$ and some real diagonal matrix $\Lambda$ of rank $r$. Of course, now some of the eigenvalues $\lambda_{k}$ may be negative, but, if we denote by $w_{k}$ the $k$-th column of $W$, by $s_{k}$ the sign of the corresponding eigenvalue $\lambda_{k}$, and define

$$
u_{k}=\sqrt{\left|\lambda_{k}\right|} w_{k},
$$

we obtain again $H=\sum_{k=1}^{r} s_{k} u_{k} u_{k}^{T}$, but now with real vectors $u_{k}$.

As a straightforward consequence of Lemma A.1, we obtain the following first-order description of the symplectic matrices $\widehat{I}(\varepsilon)$ in formula (4): 
Corollary A.2 Let $r \in\{1, \ldots, 2 n\}$ and let $\widehat{I}(\varepsilon)$ be as in formula (4) with $\operatorname{rank}(B)=r$. Then there exists an orthogonal set of $r$ nonzero vectors $u_{1}, \ldots, u_{r} \in \mathbb{C}^{2 n}$ and signs $s_{k} \in\{-1,1\}, k=1, \ldots, r$, such that

$$
\widetilde{I}(\varepsilon):=\prod_{k=1}^{r}\left(I+\varepsilon s_{k} u_{k} u_{k}^{T} J\right)=I_{2 n}+\varepsilon B+O\left(\varepsilon^{2}\right) .
$$

If $\widehat{I}(\varepsilon)$ is real, then the vectors $\left\{u_{1}, \ldots, u_{r}\right\}$ can be chosen to be real.

Hence, if we define $\widetilde{S}(\varepsilon)$ as in (47) above for the vectors $u_{k}$ and the signs $s_{k}$ in Corollary A.2, then at least $r$ eigenvalues of both $\widehat{S}(\varepsilon)$ in $(5)$ and $\widetilde{S}(\varepsilon)$ in (47) will have asymptotic expansions with the same leading term. In other words, Corollary A.2 allows us to analyze any small symplectic perturbation $\widehat{S}$ of a symplectic matrix $S$ as the effect of the consecutive application of $r$ rank one multiplicative perturbations (at least when analyzing the most likely behavior, described in Section 4). We stress that we are not describing here the set of all possible structure-preserving perturbations, but the set of all possible leading terms of structure-preserving perturbations. Since generic behavior of the perturbed eigenvalues depends on the first order perturbation matrix $B$ in (3), this will be enough for our purposes. 